\title{
The FES-assisted control for a lower limb rehabilitation robot: simulation and experiment
}

\author{
Yixiong Chen, Jin Hu, Long Peng and Zeng-guang Hou*
}

\begin{abstract}
To design a control strategy for iLeg, an exoskeleton robot developed for lower limb rehabilitation aiming at investigating the feasibility of integrating functional electrical stimulation (FES) with robot-based rehabilitation training, an FES-assisted training strategy combined with impedance control, has been proposed in this paper. Through impedance control, an active compliance of the robot is established, and the patient's voluntary effort to accomplish the training task is inspired. During the training process, the patient's related muscles are applied with FES which provides an extra assistance to the patient. The intensity of the FES is properly chosen in order to induce a desired active torque which is proportional to the voluntary effort extracted from the electromyography signals of the related muscles using back propagation neural networks. This kind of enhancement serves as a positive feedback which reminds the patient of the correct attempt to fulfill the desired motion. FES control is conducted by a combination of neural network-based feedforward controller and a PD feedback controller. Simulation conducted using Matlab and the experiment with a spinal cord injury subject and a healthy subject have shown satisfactory results which verify the feasibility of this control strategy.
\end{abstract}

Keywords: Rehabilitation robot; FES; Impedance control

\section{Introduction \\ Background and literature review}

Spinal cord injury (SCI) and stroke are the two main types of central nervous system injury which usually results in motor disorder such as paralysis and hemiparesis, cognitive dysfunction, as well as psychological problems. SCI is mainly caused by various kinds of accidents, while stroke is caused by an interruption of blood flow to the brain or an obstruction within a blood vessel in supplying blood to the brain $[1,2]$. Rehabilitation is one of the prime treatments for SCI and stroke patients, aiming to provoke the motor plasticity and to actuate these patients to relearn the use of their limbs. According to the rehabilitation theory, the resulting sensory feedback of the patient is associated with the cortical changes that can bring recovery of functional movement during the rehabilitation process so

*Correspondence: hou@compsys.ía.ac.cn

State Key Laboratory of Management and Control for Complex Systems, 95 Zhongguancun East Road, Beijing 100190, China that the functional deficits and the risk of consequential injuries can be minimized [3-6].

Traditional rehabilitation is labor-intensive and requires several therapists to manually help the patient to perform training, especially for lower limb rehabilitation. Therefore, it imposes an enormous economic burden to any country's health care system [5]. Meanwhile, there are a number of factors which have been found to contribute to faster motor recovery, all of which have not been taken full advantage of due to the lack of rehabilitation services [7]. Modern robotics technology has offered us a more convenient and efficient opportunity to carry out rehabilitation training, known as rehabilitation robot. Rehabilitation robot is a mechatronics system which could be used to replace the physical training effort of the therapists, allowing more intensive and repetitive motions [5]. Since research has identified the treatment intensity as a critical element for successful therapeutic outcomes, the robotic-assisted rehabilitation is likely to increase the training effect $[8,9]$. What is more, various kinds of sensors installed on the robot feed back the signals of interest,

\section{Springer}

(c) 2014 Chen et al.: licensee Springer. This is an Open Access article distributed under the terms of the Creative Commons Attribution License (http://creativecommons.org/licenses/by/4.0), which permits unrestricted use, distribution, and reproduction in any medium, provided the original work is properly credited. 
such as the force signal, electromyography (EMG) signal, and heart rate. Thereby, the states of the patient during training can be monitored. By measuring the force or EMG signal, it is possible for the robot to perceive the voluntary effort of the patient and to adjust the training pattern accordingly in order to actuate the participation of the patient $[10,11]$.

Several training strategies for rehabilitation robot have been presented in recent years. These control strategies can be categorized into two types. The first one is the position-based passive training which is widely used for its simplicity. However, robot based on this training strategy only moves the patient along predefined, fixed trajectories, and the guidance provided by the robot is so strong that it suppresses the voluntary motor control of the patient [12]. Therefore, this strategy is less likely to motivate the patient. The second one is the impedancebased active training. The rehabilitation robot designed for this type executes a task by amplifying human force or supplying the human operator with an assisting force $[13,14]$. Under this type of control strategy, an active compliance environment between the patient and the robot is established, while the assistant level can be adjusted by the impedance parameters. When the movement of the patient deviates from the predefined trajectory, a restoring force is generated using an appropriately designed impedance and then applied to the patient [6]. Unlike position-based training, impedance-based training is position error-tolerated, because research has emphasized that the kinematic errors generated during movement are a fundamental neural signal that drives motor adaptation [15].

On of the most commonly used technology for rehabilitation is the functional electrical stimulation (FES), which uses short electrical pulses to generate FES-induced contraction of the paralyzed muscles, and the level of the contraction can be controlled by modulating the intensity of FES [16], and the therapeutic effect of FES in rehabilitation is known to be increased when associated with a person's voluntary effort [17]. For this reason, some researchers has integrated FES into robotic-based rehabilitation to improve the training efficiency $[3,18,19]$, in which an extra actuator besides the motor of the robot is available (considering the muscle under FES as a 'soft motor'). Therefore, the robot-generated assistance can be partially compensated by the FES-induced contraction of the muscle resulting in an increase of the muscle activity. If the level of FES assistance is regulated by the voluntary effort of the patient, a higher degree of functional recovery can be expected $[3,20]$.

iLeg is a horizontal exoskeleton robot designed for lower limb rehabilitation. To develop a control strategy for iLeg, an FES-assisted training strategy combined with impedance control is introduced in this paper, aiming to achieve two goals. The first one is to create a compliance environment to inspire the voluntary effort of the patient, and the second one is to provide assistance through FES rather than purely through robot. Active compliance is guaranteed by impedance control in order to establish a soft interaction between the patient and the robot. The velocity of the movement depends on the measured voluntary effort. Basically, if no voluntary effort is measured, the rehabilitation robot only provides minimal assistance which is just sufficient to counterbalance the weight of the limb. The FES assistance, which is proportional to the patient's voluntary effort, is applied to the patient. This kind of enhancement serves as a positive feedback which reminds the patient of the correct attempt to fulfill the desired motion. To ensure that the active torque induced by FES follows the desired torque calculated from the voluntary effort, a combination of an inverse model-based feedforward and a PD feedback controller is implemented to compose the FES controller.

The rest of the paper is organized as follows. In the 'Methods' section, the rehabilitation robot is described in detail; the dynamic model of the leg-robot hybrid system is introduced and analyzed, while the model of the FES-stimulated muscle is also given. Then the control strategy of both impedance control and FES control are proposed, and the methods used for estimating muscle torques by system identification process and the method used for extracting voluntary effort by EMG signals are also described. The simulation conducted using Matlab and experimental results are depicted in the 'Results' section. Finally, conclusions are drawn in the last section.

\section{Research design and methods Methods Model description iLeg lower limb rehabilitation robot}

iLeg is a horizontal exoskeleton lower limb rehabilitation robot designed by our institute. Considering that the movement of the human lower limbs in the sagittal plane is the basic movement form, and many other lower limbs rehabilitation robots such as Locomat and MotionMaker follow this principle, iLeg is also designed with three degrees of freedom (DOF) that can realize the movement of the hip joint, the knee joint, and the ankle joint in the sagittal plane for each side. The prototype of the iLeg is showed in Figure 1.

As it can be seen in Figure 2, the electrical control system of iLeg consists of the following main modules: a host PC, a motion control subsystem, an analog signal acquisition subsystem, a safe-security subsystem, an EMG acquisition subsystem, and a functional electrical stimulator. The motion control subsystem contains motion control card, motor drivers, and DC motors for each joint of both 

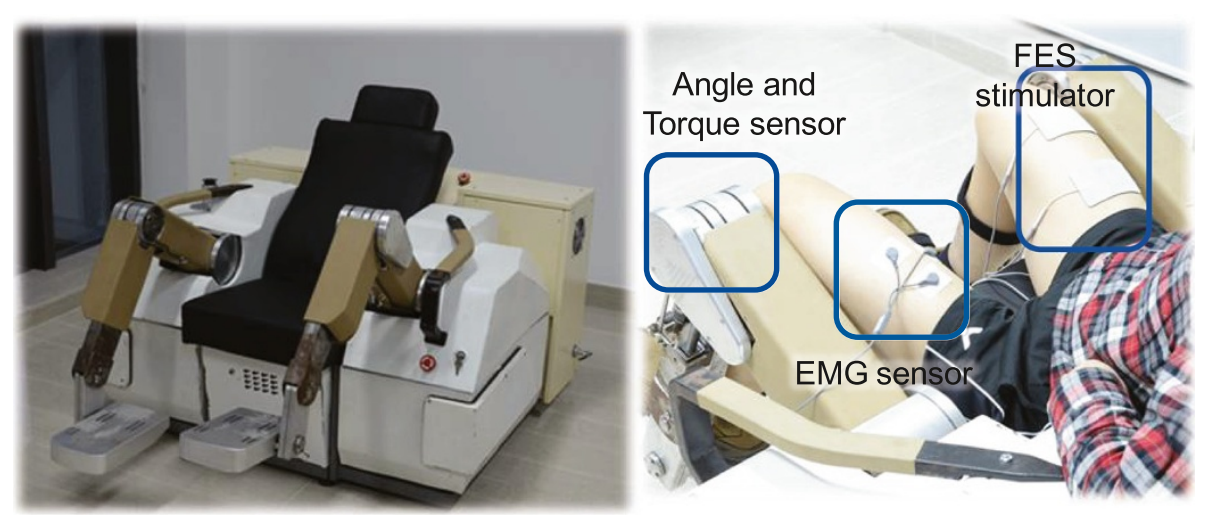

Figure 1 The prototype of iLeg lower limb rehabilitation robot.

sides. The hip joint is driven by chain after a reduction ratio of 1:120. The knee joint is driven through harmonic reducer (1:188) and a timing belt transmission. The ankle joint is driven directly through timing belt transmission. Besides, both of the thigh length and the shank length are adjustable through DC motors to ensure perfect alignment of each joint. The acquisition of the torques and the interaction force between the human leg and iLeg are carried out through analog signal acquisition subsystem. This subsystem contains torque sensors equipped at each joint and force sensor installed at the pedal. The safesecurity subsystem is responsible for monitoring whether each joint are working within their safety ranges. If a joint is exceeding the safety range, the corresponding limit switch will be triggered, and the brake of this joint will be turned on to prevent secondary damage to the patient. To extract the voluntary intention of the patient, an eight-channel self-made EMG acquisition subsystem is integrated. Since EMG signal contains most of its power in the frequency range of 5 to $500 \mathrm{~Hz}$, the sampling

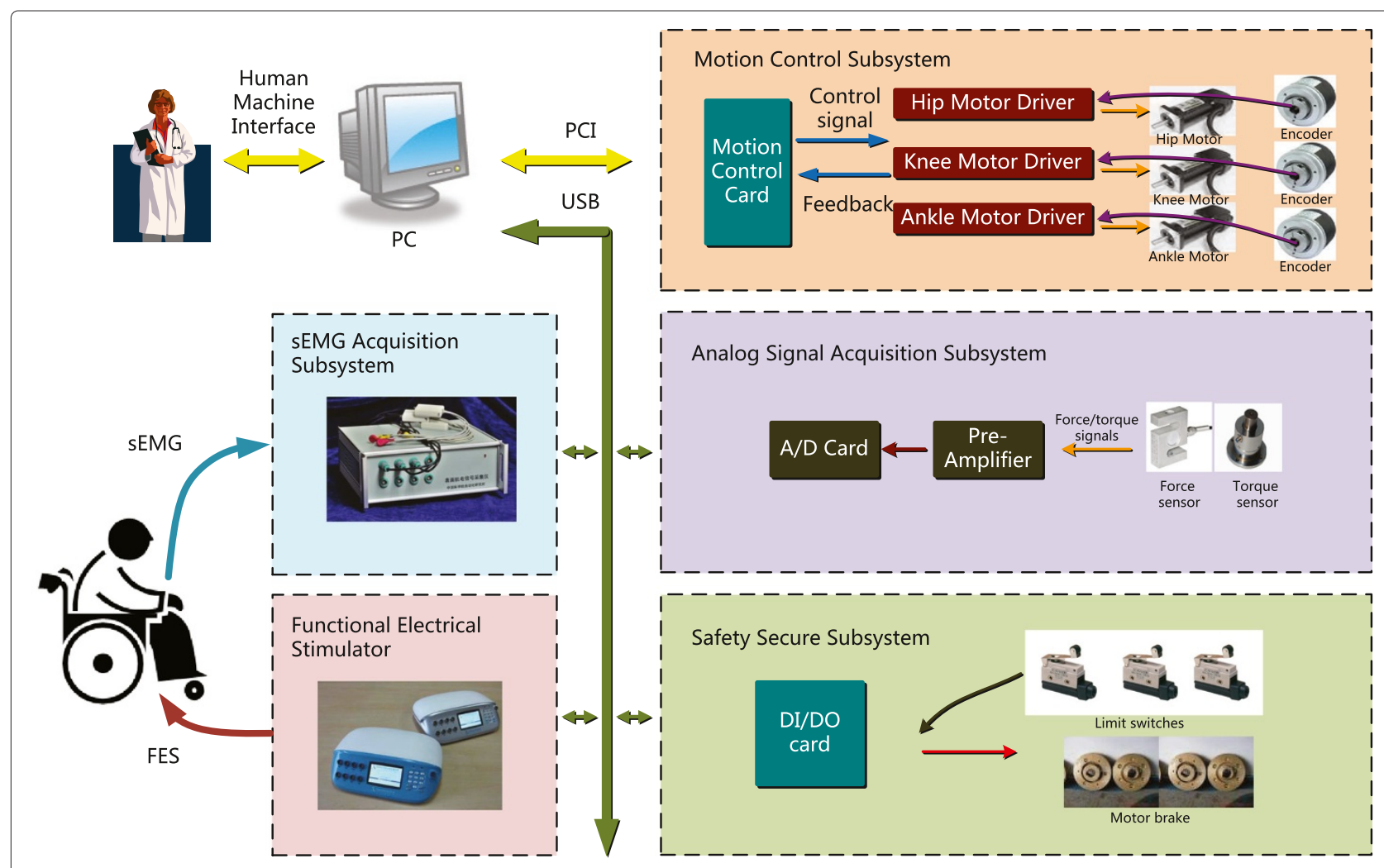

Figure 2 The electrical control system of iLeg. 
rate of each channel is set to be $2 \mathrm{kHz}$. To enhance the muscle contraction during rehabilitation training, a functional electrical stimulator is also employed in iLeg. The self-made functional electrical stimulator has eight channels and is able to apply dual-phase current pulse to the patient's muscle. Each channel of the stimulator can be controlled independently with pulse frequency of 0 to $100 \mathrm{~Hz}$, pulse width of 0 to $1,000 \mu \mathrm{s}$ and pulse amplitude of 0 to $100 \mathrm{~mA}$. All these subsystems are connected to the host $\mathrm{PC}$ whose main tasks are system management and algorithm processing via PCI bus and universal serial bus (USB).

\section{Kinematic and dynamic of human leg and iLeg}

The simplified model of human leg and iLeg is shown in Figure 3. Since the ankle joint is fix at $90^{\circ}$ during the training process and the joints of human leg and iLeg are well aligned, the leg-robot system can be considered as two parallel two-link plants. The patient's foot is fastened to the pedal, and the physical interaction occurs at the endpoint. Gluteus maximus and quadriceps femoris are the two muscle groups stimulated by FES to generate active torques on the hip joint and the knee joint. In Figure $3, q_{\mathrm{i}}$ is the angle of joint $i ; m_{\mathrm{i}}^{\mathrm{r}}, m_{\mathrm{i}}^{\mathrm{h}}$, and $l_{\mathrm{i}}$ represent the mass of the robot, the mass of the leg, and the length of link $i$, respectively. $F_{\mathrm{r} 2 \mathrm{~h}}$ and $F_{\mathrm{h} 2 \mathrm{r}}$ denote the interaction forces between the robot and the human leg. The forward kinematics of the system can be calculated by the following:

$$
X=\left\{\begin{array}{l}
x \\
y
\end{array}\right\}=\left\{\begin{array}{c}
l_{1} \cos q_{1}+l_{2} \cos \left(q_{1}+q_{2}\right) \\
l_{1} \sin q_{1}+l_{2} \sin \left(q_{1}+q_{2}\right)
\end{array}\right\}
$$

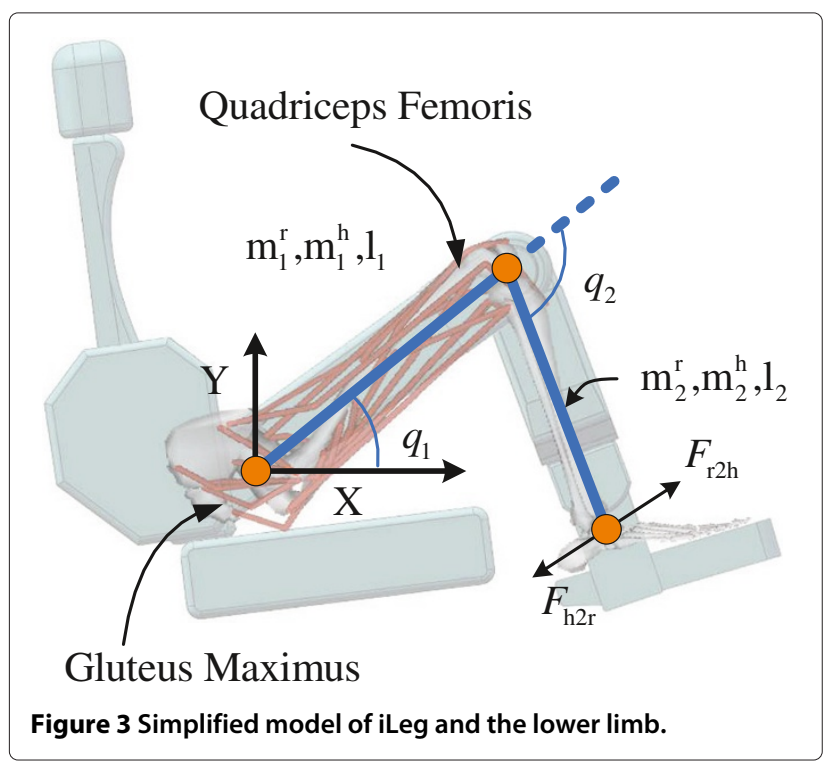

Accordingly, the speed of the end-point $\dot{X}$ in the Cartesian coordinate is determined by the differential kinematics equation:

$$
\dot{X}=J \dot{q}
$$

where $\dot{q}$ is the angular velocity in the joint space, and $J$ is the Jacobian matrix represented as follows:

$$
J=\left[\begin{array}{cc}
-l_{1} \sin q_{1}-l_{2} \sin \left(q_{1}+q_{2}\right) & -l_{2} \sin \left(q_{1}+q_{2}\right) \\
l_{1} \cos q_{1}+l_{2} \cos \left(q_{1}+q_{2}\right) & l_{2} \cos \left(q_{1}+q_{2}\right)
\end{array}\right]
$$

Inverse kinematics of the simplified model can be derived from the forward kinematics equation and expressed as follows:

$$
q=\left\{\begin{array}{l}
q_{1} \\
q_{2}
\end{array}\right\}=\left\{\begin{array}{c}
\tan ^{-1} \frac{y}{x}+\cos ^{-1}\left(\frac{y^{2}+x^{2}+l_{1}^{2}-l_{2}^{2}}{2 l_{1} \sqrt{y^{2}+x^{2}}}\right) \\
-\cos ^{-1}\left(\frac{y^{2}+x^{2}-l_{1}^{2}-l_{2}^{2}}{2 l_{1} l_{2}}\right)
\end{array}\right\}
$$

In generalized form, the dynamic model of the robot system is expressed by the following:

$$
M_{\mathrm{r}}(q) \ddot{q}+C_{\mathrm{r}}(q, \dot{q}) \dot{q}+G_{\mathrm{r}}(q)+\tau_{\mathrm{rv}}(\dot{q})+\tau_{\mathrm{rf}}(\dot{q})=\tau_{\mathrm{r}}-\tau_{\mathrm{h} 2 \mathrm{r}}
$$

where $\tau_{\mathrm{rv}}(\dot{q})=k_{\mathrm{vis}} \dot{q}$ and $\tau_{\mathrm{rf}}(\dot{q})=k_{\text {fri }} \operatorname{sgn}(\dot{q})$ represent the viscosity and friction of the robot; $\tau_{\mathrm{h} 2 \mathrm{r}}$ represents the external torque exert by human; $M(q), C(q, \dot{q})$, and $G(q)$ are the inertia, Coriolis/centripetal, and gravity matrixes given as

$$
\begin{aligned}
& M_{1,1}^{\mathrm{r}}=\left(\frac{1}{3} m_{1}^{\mathrm{r}}+m_{2}^{\mathrm{r}}\right) l_{1}^{2}+\frac{1}{3} m_{2}^{\mathrm{r}} l_{2}^{2}+m_{2}^{\mathrm{r}} l_{1} l_{2} \cos q_{2}, \\
& M_{1,2}^{\mathrm{r}}=M_{2,1}^{\mathrm{r}}=\frac{1}{3} m_{2}^{\mathrm{r}} l_{2}^{2}+\frac{1}{2} m_{2}^{\mathrm{r}} l_{1} l_{2} \cos q_{2}, M_{2,2}^{\mathrm{r}}=\frac{1}{3} m_{2}^{\mathrm{r}} l_{2}^{2}, \\
& C_{1,1}^{\mathrm{r}}=-\frac{1}{2} m_{2}^{\mathrm{r}} l_{1} l_{2} \dot{q}_{2} \sin q_{2}, C_{2,1}^{\mathrm{r}}=\frac{1}{2} m_{2}^{\mathrm{r}} l_{1} l_{2} \dot{q}_{1} \sin q_{2}, \\
& C_{1,2}^{\mathrm{r}}=-\frac{1}{2} m_{2}^{\mathrm{r}} l_{1} l_{2}\left(\dot{q}_{1}+\dot{q}_{2}\right) \sin q_{2}, C_{2,2}^{\mathrm{r}}=0, \\
& G_{1,1}^{\mathrm{r}}=\left(\frac{1}{2} m_{1}^{\mathrm{r}}+m_{2}^{\mathrm{r}}\right) g l_{1} \cos q_{1}+\frac{1}{2} m_{2}^{\mathrm{r}} g l_{2} \cos \left(q_{1}+q_{2}\right), \\
& G_{2,1}^{\mathrm{r}}=\frac{1}{2} m_{2}^{\mathrm{r}} g l_{2} \cos \left(q_{1}+q_{2}\right)
\end{aligned}
$$

where $m_{i}^{\mathrm{r}}, l_{\mathrm{i}}$ represent the mass and the length of link $i$. Similar to the robot dynamic, the dynamic model of human leg can be described as

$$
M_{\mathrm{h}}(q) \ddot{q}+C_{\mathrm{h}}(q, \dot{q}) \dot{q}+G_{\mathrm{h}}(q)+\tau_{\mathrm{hv}}(\dot{q})+\tau_{\mathrm{he}}(q)=\tau_{\mathrm{r} 2 \mathrm{~h}}+\tau_{\mathrm{m}}
$$

where $M_{\mathrm{h}}(q), C_{\mathrm{h}}(q, \dot{q})$, and $G_{\mathrm{h}}(q)$ are the inertia matrix, the Coriolis/centripetal matrix, and the gravity vector of human leg, respectively. Their expressions are similar to 
those in the robot dynamic, but the only difference is that the masses of each robot link in (6) is replaced by the masses of human' thigh and shank. $\tau_{\text {hv }}(\dot{q})$ and $\tau_{\text {he }}(q)$ describe the passive viscous and passive elastic characters of the lower limb, which are described in detail in [21]. Unlike robot dynamics, the human leg can be considered as driven by redundant actuators (muscle contraction and robot assistance). The muscle contraction contributes to $\tau_{\mathrm{m}}$ in Equation 7, while $\tau_{\mathrm{r} 2 \mathrm{~h}}$ represents the assistance from the robot, which can be measured by the force sensor attached on the end effector using the following equation:

$$
\tau_{\mathrm{r} 2 \mathrm{~h}}=J^{\mathrm{T}} F_{\mathrm{r} 2 \mathrm{~h}}
$$

where $F_{\mathrm{r} 2 \mathrm{~h}}$ is the force imposed to the leg due to interaction between human and robot, and $J$ is the Jacobian matrix. By adding Equations 7 and 5, we can obtain the dynamic model of the leg-robot hybrid system which is represented as

$$
M(q) \ddot{q}+C(q, \dot{q}) \dot{q}+G(q)+\tau_{\mathrm{pas}}(q, \dot{q})=\tau_{\mathrm{r}}+\tau_{\mathrm{m}}
$$

where $\tau_{\text {pas }}=\tau_{\mathrm{hv}}(\dot{q})+\tau_{\mathrm{he}}(q)+\tau_{\mathrm{rv}}(\dot{q})+\tau_{\mathrm{rf}}(\dot{q})$, and $M_{\mathrm{h}}(q)$, $C_{\mathrm{h}}(q, \dot{q})$ and $G_{\mathrm{h}}(q)$ are the inertia matrix, the Coriolis/centripetal matrix, and the gravity vector of the hybrid system, respectively. According to Equation 9, there are two types of inputs which contribute to the input torque of the system: the one from DC motor and the one from the muscle contraction.

\section{FES-stimulated skeletal muscle}

During the training stage, two human muscles (gluteus maximus and quadriceps femoris) will be stimulated to produce partial torques around the hip joint and the knee joint. Therefore, a biological model is needed to depict the muscle response to FES, i.e., how the FES delivered to the relative muscle is converted into an active moment at the joint. Figure 4 shows a biological model of FES-stimulated muscle developed by Doctor Riener which is used in this study $[22,23]$. The active torque produced by the FESinduced muscle contraction is described as two parts, namely, activation dynamics and contraction dynamics, while the passive torque of the muscle is described as passive elastic and passive viscous properties [22]. Note that the original model has two inputs for activation dynamics which are stimulation pulse width and frequency; however, for the sake of simplicity, we fix the stimulation frequency for each muscle constantly at $50 \mathrm{~Hz}$ while regulating the intensity of FES by controlling the pulse width of the stimulation using FES controller.

The active torque of FES-induced muscle contraction is the product of contraction dynamics and activation dynamics given as

$$
\tau_{\text {fes }}=a_{\text {act }} f_{\text {fv }} f_{\mathrm{fl}} F_{\text {max }} \text { ma }
$$

In the contraction dynamics, four parts are involved, i.e., moment arm, ma, maximum isometric force $F_{\max }$, forcelength relation $f_{\mathrm{fl}}$, and force-velocity relation $f_{\mathrm{fv}}$. The product of these four parts denotes the maximum torque of the stimulated muscle at certain joint angle and angular velocity. The force-length relation is calculated using

$$
f_{\mathrm{fl}}=\exp \left\{-[(\bar{l}-1) / \varepsilon]^{2}\right\}
$$

where $\bar{l}$ is the muscle length normalized with respect to the optimal muscle length opt, and $\varepsilon$ is a shape factor. The force-velocity relation is determined by

$$
f_{\mathrm{fv}}=0.54 \arctan (5.69 \bar{v}+0.51)+0.745
$$

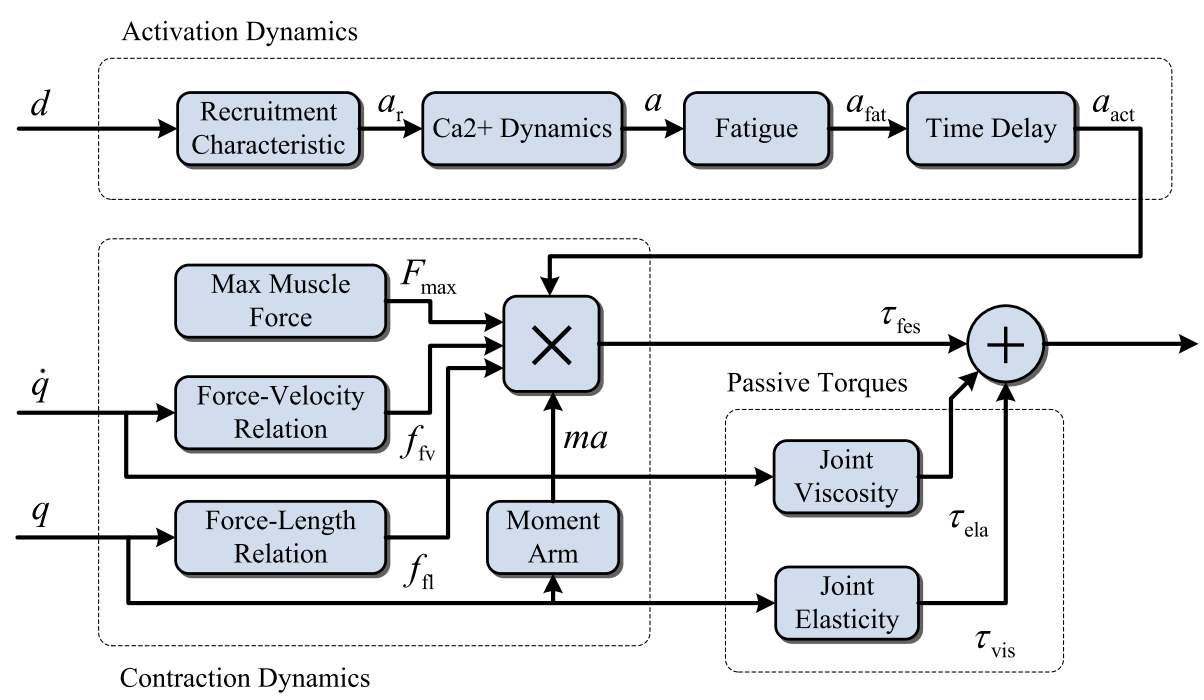

Figure 4 Activation dynamic and contraction dynamic of the muscle. 
where $\bar{v}$ is the muscle velocity normalized with respect to the maximum contraction velocity $v_{\mathrm{m}}$ of the muscle. The muscle length and velocity can be calculated using joint angle, angular velocity, and moment arm of the muscle described in detail in [22]. The activation dynamics determines how the motion unit is recruited by FES in the final form of $a_{\text {act }}$. Basically, the activation dynamics is composed of four segments. They are the recruitment characteristic, the Calcium dynamic, the fatigue process, and a delay of about $25 \mathrm{~ms}$. The recruitment characteristic shows the relationship between the FES pulse width and the percentage of the motor unit activated by FES, and it is given as

$$
\begin{aligned}
a_{\mathrm{r}}= & a_{\mathrm{f}}\left\{c _ { 1 } \left\{\left(d-d_{\mathrm{thr}}\right) \arctan \left[k_{\mathrm{thr}}\left(d-d_{\mathrm{thr}}\right)\right]\right.\right. \\
& \left.\left.-\left(d-d_{\mathrm{sat}}\right) \arctan \left[k_{\mathrm{sat}}\left(d-d_{\mathrm{sat}}\right)\right]\right\}+c_{2}\right\}
\end{aligned}
$$

where $d$ is the pulse width of FES, $d_{\text {thr }}$ and $d_{\text {sat }}$ denote pulse width values corresponding to threshold and saturation. The shape of the recruitment curve is describe using $c_{1}, c_{2}, k_{\mathrm{thr}}$, and $k_{\mathrm{sat}} . a_{\mathrm{f}}$ is introduced to represent the frequency characteristic which is a function of the stimulation frequency in [22]. Since the stimulation frequency is fixed at $50 \mathrm{~Hz}$, here $a_{\mathrm{f}}$ is a constant. Calcium dynamics presents the mechanism that calcium ion released from sarcoplasmic reticulum and is expressed using the following two-order linear relation:

$$
T_{\mathrm{Ca}}^{2} \ddot{a}+2 T_{\mathrm{Ca}} \dot{a}+a=a_{\mathrm{r}}
$$

where $T_{\mathrm{Ca}}$ is the time constant, and $a$ denotes non-fatigue muscle activation. Fitness function is introduced to depict the effect of muscle fatigue and recovery phenomenon using the following first-order relation:

$$
\frac{d \mathrm{fit}}{d t}=\frac{0.55 a\left(\mathrm{fit}_{\min }-\mathrm{fit}\right)}{T_{\mathrm{fat}}}+\frac{(1-\mathrm{fit})(1-0.55 a)}{T_{\mathrm{rec}}}
$$

where fit is the fitness of the muscle, while fit ${ }_{\min }$ is the minimum fitness. Time constants for fatigue and recovery are given as $T_{\text {fat }}$ and $T_{\text {rec }}$. Final activation $a_{\text {act }}$ is the product of fitness fit and non-fatigue activation $a$ with a constant time delay $T_{\mathrm{del}}$. After the maximum torque of the stimulated muscle at certain joint angle and angular velocity are being scaled by the activation $a_{\text {act }}$, the active torque of the stimulated muscle can be obtained.

\section{Control strategy}

The control strategy contains two main parts, namely, the impedance control and FES control. The goal of the first one is to achieve certain desired active compliance on the robot, while the goal of the second one is to generate desired FES-induced torques around the joint by regulating the FES intensity. The architecture of the control strategy is shown in Figure 5.

\section{Impedance control}

The aim of an impedance controller is to establish a massdamper-spring relationship between the position and the force so that an active compliance between the robot and the leg can be achieved [24-26]. The desired impedance relationship can be expressed as follows:

$$
M_{\mathrm{d}}\left(\ddot{x}-\ddot{x}_{\mathrm{d}}\right)+B_{\mathrm{d}}\left(\dot{x}-\dot{x}_{\mathrm{d}}\right)+K_{\mathrm{d}}\left(x-x_{\mathrm{d}}\right)=F_{\text {ext }}
$$

where $M_{\mathrm{d}}, B_{\mathrm{d}}, K_{\mathrm{d}}$ are positive definite matrices representing the desired inertia, damping, and stiffness of the system, respectively, and $\ddot{x}_{\mathrm{d}}, \dot{x}_{\mathrm{d}}, x_{\mathrm{d}}$ are the references for the acceleration, the velocity, and the position of the endeffector in the Cartesian space, while $\ddot{x}, \dot{x}$ and $x$ stand for the actual acceleration, velocity and position, respectively. $F_{\text {ext }}$ is the external force imposed on the end effector. In the case of our rehabilitation robot, $F_{\text {ext }}$ means $F_{\mathrm{m}}$ in Figure 5, which is the force produced by muscle contraction, either by voluntary-controlled contraction or by FES-induced contraction. Usually, the desired impedance is chosen to be a second-order linear equation, as in massspring-damper system; however, in majority of the kinds of rehabilitation exercises, the speed of the robot is relatively low, so the acceleration effect can be neglected $[27,28]$, resulting Equation 16 to be degenerated to the following equation:

$$
B_{\mathrm{d}}\left(\dot{x}-\dot{x}_{\mathrm{d}}\right)+K_{\mathrm{d}}\left(x-x_{\mathrm{d}}\right)=F_{\mathrm{m}}
$$

If the manipulator follows a speed reference given by

$$
\dot{x}_{\text {ref }}=\dot{x}_{\mathrm{d}}+B_{\mathrm{d}}^{-1}\left[F_{\mathrm{m}}-K_{\mathrm{d}}\left(x-x_{\mathrm{d}}\right)\right]
$$

the rehabilitation robot will behave as described by Equation 17. The speed tracking task is achieved by a PI speed controller in the joint space given as

$$
\tau_{\mathrm{PI}}=K_{\mathrm{P}}\left(\dot{q}_{\text {ref }}-\dot{q}\right)+K_{\mathrm{I}} \int\left(\dot{q}_{\text {ref }}-\dot{q}\right) \mathrm{d} t
$$

where, $K_{\mathrm{P}}$ and $K_{\mathrm{I}}$ are the proportional and integral gains of the speed controller. $\dot{q}_{\text {ref }}$ is the reference of the angular velocity given by $\dot{q}_{\text {ref }}=J^{-1} \dot{x}_{\text {ref }}$, where $J$ represents the Jacobian matrix.

The impedance control strategy is conducted in a leg press exercise, in which a predefined trajectory of the endpoint (the ankle joint of the patient) is predefined. The patient is encouraged to complete a leg press motion and is also expected to maintain his ankle on the predefined trajectory during his effort. $\dot{q}_{\mathrm{d}}$ is set to be zero in this control, so basically the rehabilitation robot behaves as a gravity canceler. If there is no muscular force measured, the robot does not assist the patient to move towards the target point. $x_{\mathrm{d}}$ in Equation 18 is the desired position for the endpoint on the predefined trajectory at a specific time point, and $x_{\mathrm{d}}$ is moving towards the target point at 


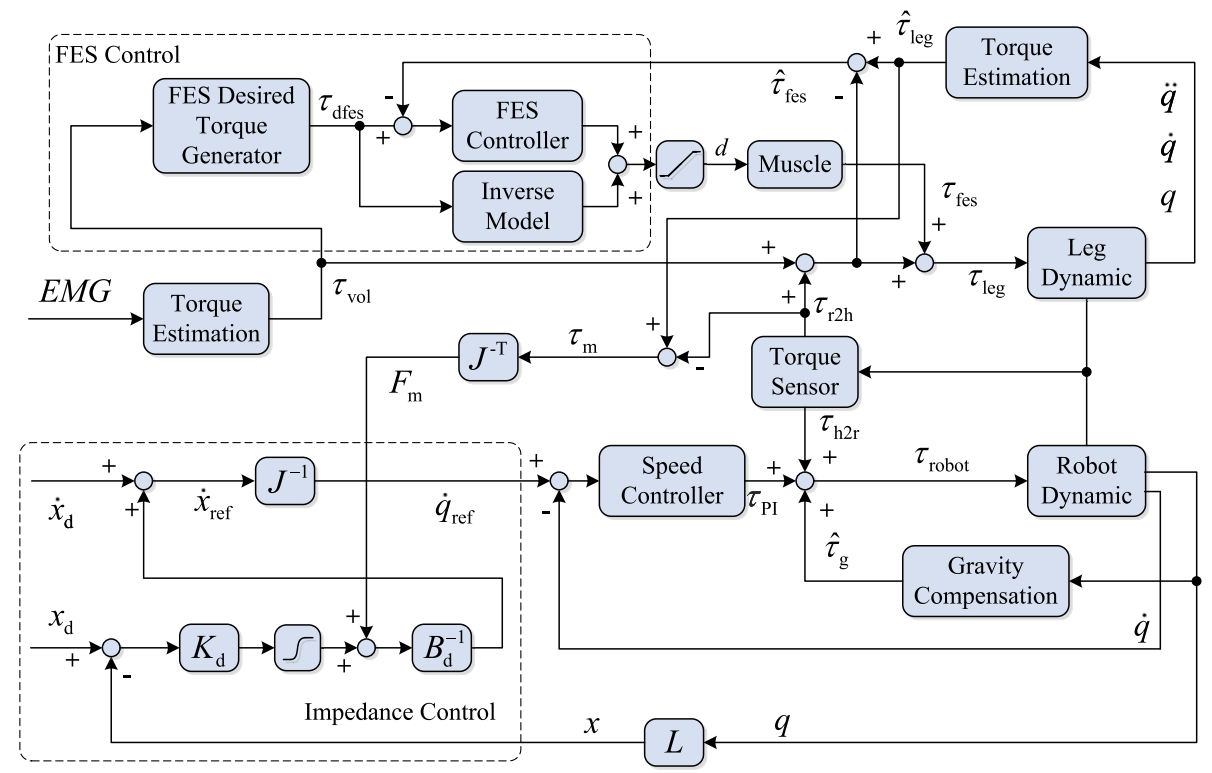

Figure 5 Control architecture of FES-assisted training strategy.

a constant speed, meaning a 'virtual moving wall' which is pushing the patient towards the target [12]. A waiting window for delayed assistance is also employed, embodied by adding a modification factor to the stiffness component of the impedance control described in Equation 17. The factor mod is given by a sigmoid function expressed as follows:

$$
\bmod =\frac{1}{1+e^{-a \cdot \operatorname{dis}+b}}
$$

where dis represents the distance between the actual and desired position at a specific time point; $a, b$ define the shape of the sigmoid function. The task is error-tolerated, but if the endpoint deviates too far from the desired position, the patient will feel harder to maintain the incorrect effort, so he has to make a proper adjustment in order to accomplish the task. From the perspective of the patient, this impedance control provides a virtual channel with the predefined trajectory in its center, in which the motion is partially constrained in the desired direction.

\section{FES control}

Since the patient has insufficient voluntary contraction of his muscle, FES is implemented to enhance the contraction, aiming to assist the patient to accomplish the leg press task. To be more specific, the desired FES-induced force is proportional to the decomposed voluntary force whose direction is alongside with the direction of the predefined trajectory. Because this part of the voluntary force is considered as the 'correct' effort for the task, the decomposed voluntary force which is vertical to the predefined path is considered as the 'incorrect' effort and will not be enhanced. The voluntary force $F_{\mathrm{vol}}$ can be transferred from the voluntary contraction torque $\tau_{\mathrm{vol}}$, which is estimated by the EMG signals of the related muscle groups. The calculation of the desired FES-induced torque $\tau_{\text {dfes }}$ is given as

$$
\tau_{\mathrm{dfes}}=J^{\mathrm{T}}\left[\begin{array}{cc}
\cos \theta & \sin \theta \\
-\sin \theta & \cos \theta
\end{array}\right] J^{-\mathrm{T}} \tau_{\mathrm{vol}}
$$

where $J$ is the Jacobian matrix, and $\theta$ represents the angle of $F_{\mathrm{vol}}$ to the predefined trajectory.

As shown in Figure 5, the FES torque control is achieved with the combination of a feedforward controller and a feedback controller. An inverse model of the stimulated muscle and a PD controller are employed and serve as the feedforward controller and the feedback controller, respectively. The advantage of using the combination of these two controllers compared with only using one of them is significant. The delay and highly nonlinear characters of muscle response to FES are easy to cause oscillation when the FES is regulated merely by the feedback controller. On the other hand, the feedforward controller is incapable of dealing with disturbance and eliminating error [23,29].

A three-layer neural network is introduced to capture the nonlinear relation of the inverse model. For each stimulated muscle (gluteus maximus and quadriceps femoris), a three-layer back propagation (BP) neural network is constructed whose structure is shown in Figure 6. Since the inverse model of muscle requires joint angle, joint angular velocity, and desired torque to be the input, the 


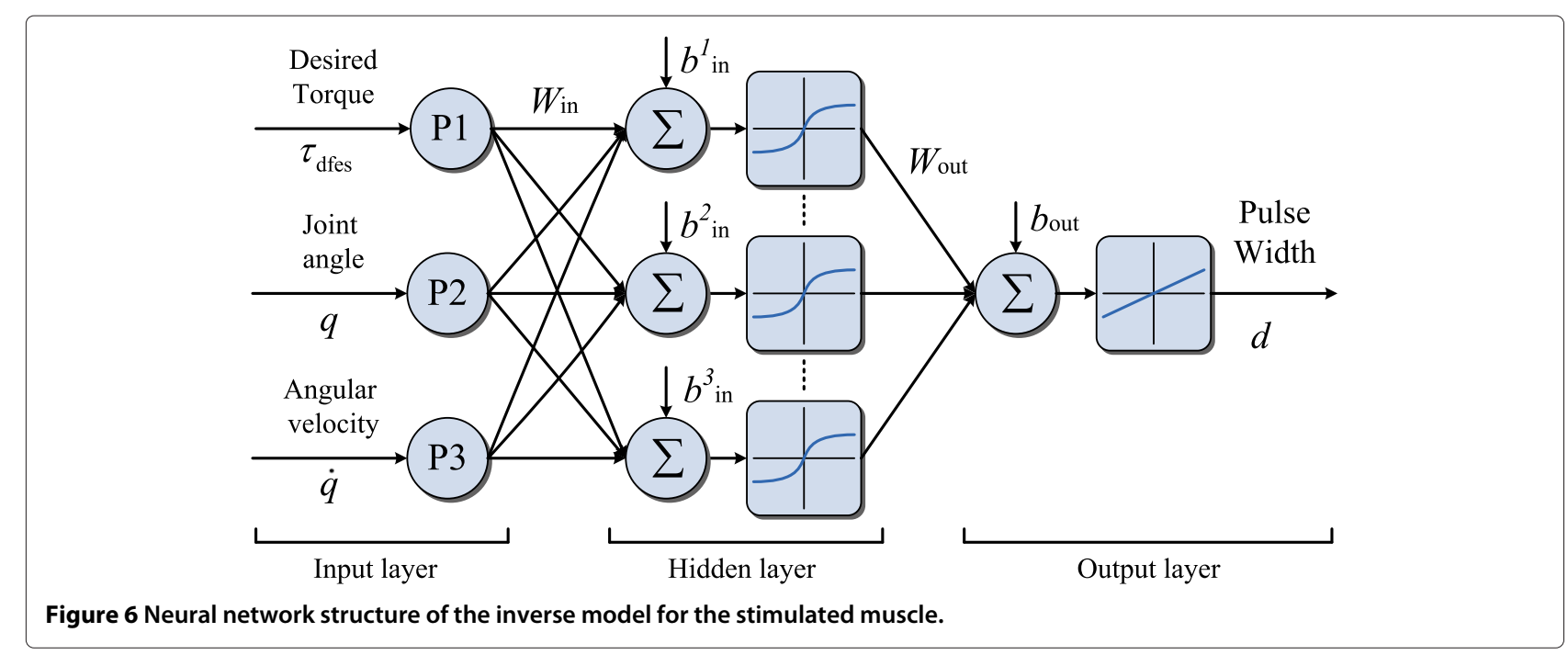

first layer of neural network consists of three input neurons. The neuron in the output layer calculates the pulse width of the FES with the linear function. The weight matrixes between the input layer, the hidden layer, and the output layer are adjusted using back propagation algorithm.

The PD controller which serves as the feedback controller is defined as follows:

$$
d=K_{\mathrm{P}} e+K_{\mathrm{D}} \dot{e}, \quad e=\tau_{\mathrm{dfes}}-\tau_{\mathrm{fes}}
$$

where $d$ is the pulse width of the FES, and $K_{\mathrm{P}}, K_{\mathrm{D}}$ are proportional and derivative gains of the feedback controller, respectively. $e$ represents the error between desired torque calculated in (12) and the actual torque induced by FES.

\section{FES-induced torque estimation}

In the case of applying FES to a moving limb, it is hard to measure the actual active torque $\tau_{\text {fes }}$ induced by FES directly. So an estimation of $\tau_{\text {fes }}$ should be made. The total input torque of the leg-robot hybrid system which is the right side of Equation 9 can be rewritten as

$$
\tau_{\text {total }}=\tau_{\mathrm{r}}+\tau_{\mathrm{m}}=\tau_{\mathrm{r}}+\tau_{\mathrm{fes}}+\tau_{\mathrm{vol}}
$$

That is to say, the torque caused by muscle contraction $\tau_{\mathrm{m}}$ is considered as a combination of two parts: the one from voluntary-controlled contraction $\tau_{\text {vol }}$ and the one from FES-induced contraction $\tau_{\text {fes }}$. Therefore, to acquire the value of $\tau_{\text {fes }}$, the other three items have to be obtained. $\tau_{\mathrm{r}}$ can be measured via torque sensors, and $\tau_{\mathrm{vol}}$ can be predicted by the EMG signal. As for $\tau_{\text {total }}$, an system identification process is adopted so that the estimation of $\tau_{\text {fes }}$ can be calculated from

$$
\tau_{\mathrm{fes}}=\tau_{\mathrm{total}}-\tau_{\mathrm{r}}-\tau_{\mathrm{vol}}
$$

\section{Hybrid-activated torque}

The first step to obtain the FES-induced torque is to calculate the muscle torque $\tau_{\mathrm{m}}$ which is produced by muscle contraction either it is FES-induced or voluntarycontrolled, so $\tau_{\mathrm{m}}$ is considered as hybrid-activated torque. A system identification process is introduced to make an estimation of this hybrid-activated torque. We rewrite Equation 9 as

$$
D(q, \dot{q}, \ddot{q}) \chi=\tau_{\mathrm{r}}+\tau_{\mathrm{m}}
$$

where $D(q, \dot{q}, \ddot{q})$ is the $2 \times 8$ regression matrix associated with the $8 \times 1$ dynamic parameters $\chi$. For the identification process, no force is applied on the robot [30], thus, $\tau_{\mathrm{m}}=0$ and

$$
D(q, \dot{q}, \ddot{q}) \chi=\tau_{\mathrm{r}}
$$

$D(q, \dot{q}, \ddot{q})$ is given as

$$
\begin{aligned}
D_{11}= & \ddot{q}_{1}, D_{12}=g \cos \left(q_{1}\right), D_{13}=\ddot{q}_{2}, \\
D_{14}= & l_{1}\left(2 \ddot{q}_{1}+\ddot{q}_{2}\right) \cos \left(q_{2}\right)-l_{1}\left(\dot{q}_{2}^{2}+2 \dot{q}_{1} \dot{q}_{2}\right) \sin \left(q_{2}\right) \\
& +g \cos \left(q_{1}+q_{2}\right), \\
D_{15}= & \operatorname{sgn}\left(\dot{q}_{1}\right), D_{16}=0, D_{17}=\dot{q}_{1}, D_{18}=0, \\
D_{21}= & 0, D_{22}=0, D_{23}=\ddot{q}_{1}+\ddot{q}_{2}, \\
D_{24}= & l_{1} \ddot{q}_{1} \cos \left(q_{2}\right)+l_{1} \dot{q}_{1}^{2} \sin \left(q_{2}\right)+g \cos \left(q_{1}+q_{2}\right), \\
D_{25}= & 0, D_{26}=\operatorname{sgn}\left(\dot{q}_{2}\right), D_{27}=0, D_{28}=\dot{q}_{2}
\end{aligned}
$$

and $\chi$ is defined as

$$
\begin{aligned}
& \chi_{1}=I_{1}+I_{2}+l_{1}^{2} m_{2}, \chi_{2}=l_{1}\left(m_{2}+m_{1} / 2\right), \chi_{3}=I_{2}, \\
& \chi_{4}=m_{2} l_{2} / 2, \chi_{5}=k_{\mathrm{sf} 1}, \chi_{6}=k_{\mathrm{sf} 2}, \chi_{7}=k_{\mathrm{df} 1}, \chi_{8}=k_{\mathrm{df} 2}
\end{aligned}
$$

where $I_{i}$ is the inertia moment of the $i$ th link; $k_{\mathrm{sfi}}$ and $k_{\mathrm{dfi}}$ are the Coulomb and viscous friction of the $i$ th joint. 


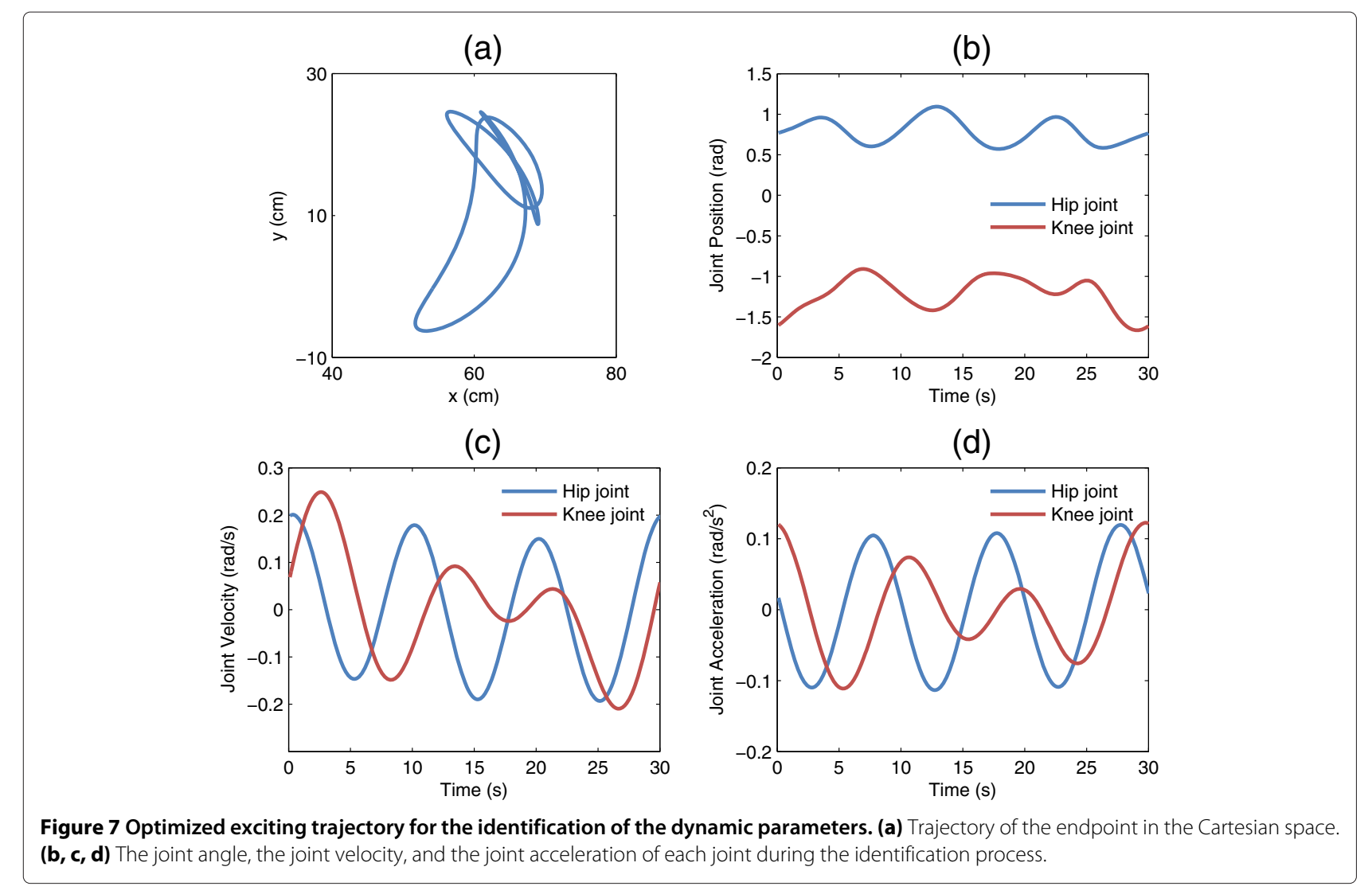

For identification, the motion states $(q, \dot{q}, \ddot{q}$ of each joint) and the torques measured by sensors should be obtained during the robot running on an exciting trajectory. Samplings are carried out at the time of $t_{1}, t_{2} \ldots t_{n}$ so that an overdetermined equation is constructed as

$$
W \chi=\Gamma
$$

where $W$ is the observation matrix defined as

$$
W=\left[\begin{array}{c}
D\left(q\left(t_{1}\right), \dot{q}\left(t_{1}\right), \ddot{q}\left(t_{1}\right)\right) \\
D\left(q\left(t_{2}\right), \dot{q}\left(t_{2}\right), \ddot{q}\left(t_{2}\right)\right) \\
\vdots \\
D\left(q\left(t_{n}\right), \dot{q}\left(t_{n}\right), \ddot{q}\left(t_{n}\right)\right)
\end{array}\right]
$$

and

$$
\Gamma=\left[\tau_{\mathrm{r}}\left(t_{1}\right)^{\mathrm{T}} \tau_{\mathrm{r}}\left(t_{2}\right)^{\mathrm{T}} \ldots \tau_{\mathrm{r}}\left(t_{n}\right)^{\mathrm{T}}\right]^{\mathrm{T}}
$$

Thus, the dynamic parameters $\chi$ can be estimated using least square estimation method given by

$$
\chi=\left(W^{\mathrm{T}} W\right)^{-1} W^{\mathrm{T}} \Gamma
$$

To improve the accuracy of the estimation, the exciting trajectory should be optimized for the sufficient excitation of the hybrid system. In this study, finite Fourier series (FFS) is introduced to parameterized the exciting trajectory [31]. For each joint, the position at time $t$ is a sum of sine waves expressed as

$$
q_{i}(t)=\sum_{l=1}^{N}\left(\frac{a_{l i}}{\omega_{\mathrm{f}} l} \sin \left(\omega_{\mathrm{f}} l t\right)-\frac{b_{l i}}{\omega_{\mathrm{f}} l} \cos \left(\omega_{\mathrm{f}} l t\right)\right)+q_{i 0}
$$

where $\omega_{\mathrm{f}}$ defines the period of the exciting trajectory; $N$ is the number of FFS; $q_{i 0}$ is the initial position of the

\section{Table 1 Identification results}

\begin{tabular}{llcccccc}
\hline & Parameter & Value & Unit & & Parameter & Value & Unit \\
\hline$\chi_{1}$ & $I_{1}+l_{2}+l_{1} m_{2}$ & 57.3328 & $\mathrm{~kg} \mathrm{~m}^{2}$ & $\chi_{5}$ & $k_{s f 1}$ & -1.1060 & $\mathrm{Nm}$ \\
$\chi_{2}$ & $I_{1}\left(m_{2}+m_{1} / 2\right)$ & 2.6204 & $\mathrm{~kg} \mathrm{~m}$ & $\chi_{6}$ & $k_{s f 2}$ & 0.1335 & $\mathrm{Nm}$ \\
$\chi_{3}$ & $I_{2}$ & 6.3912 & $\mathrm{~kg} \mathrm{~m}{ }^{2}$ & $\chi_{7}$ & $k_{d f 1}$ & -4.0587 & $\mathrm{Nm} \mathrm{s} / \mathrm{rad}$ \\
$\chi_{4}$ & $m_{2} l_{2} / 2$ & 5.2661 & $\mathrm{~kg} \mathrm{~m}$ & $\chi_{8}$ & $k_{d f 2}$ & 2.5287 & $\mathrm{Nm} \mathrm{s} / \mathrm{rad}$ \\
\hline
\end{tabular}



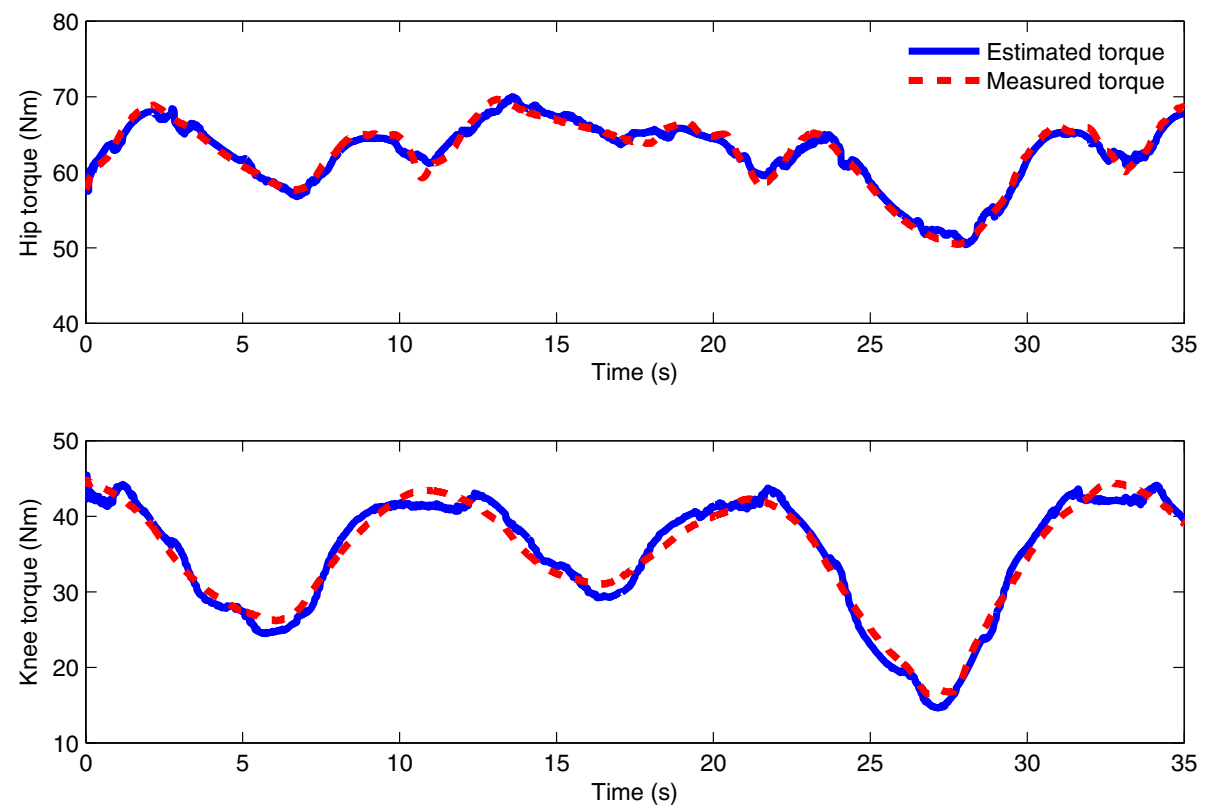

Figure 8 Measured torques and estimated torques of each joint during the validation process. Top, hip torque. Bottom, knee torque.

$i$ th joint. The optimization of the exciting trajectory is carried out by choosing the proper $a_{l i}, b_{l i}$ for each joint so that the condition number of the observation matrix can be minimized. The condition number of the observation matrix represents the upper bound for input/output error. It directly affects the convergence rate and noise immunity of the identification experiment [32]. This optimization problem can be solved using genetic algorithm (GA). During the GA optimization, variables $a_{l i}, b_{l i}$ are the decision variables which meet the constrain that the trajectory defined by them should not exceed the workspace of the robot, and the condition number of the observation matrix is chosen as the fitness function. The optimized exciting trajectory is shown in Figure 7.

The samples used for the identification are obtained by performing the exciting trajectory with the patient involved in the pre-training stage. The subject of this study is an SCI patient who has been injured in T11 for 16 months. The exciting trajectory is performed for five times. According to the method described above, the estimation of the dynamic parameters of the leg-robot hybrid system is achieved, which are given in Table 1.

An unoptimized FFS trajectory is utilized to validate the identification results. The comparison between the

\section{Table 2 The RMS error of the estimation torque ( $\mathrm{Nm}$ )}

\begin{tabular}{lcc}
\hline & Hip & Knee \\
\hline Exiting trajectory & 0.4057 & 0.3168 \\
Validation trajectory & 0.8716 & 0.5483 \\
\hline
\end{tabular}

measured torques and the estimated torques is shown in Figure 8. Root mean square (RMS) error of the estimation torques for both exiting trajectory and validation trajectory is given in Table 2. Both Figure 8 and Table 2 have shown satisfactory results which prove that the proposed method is capable of estimating the muscle torques of the patient.

\section{Voluntary torque}

The second step to obtain the FES-induced torque is to calculate the voluntary-controlled muscle torque $\tau_{\mathrm{vol}}$. EMG signal is a weak electrical potential generated by the muscle cells when these cells are activated [33], so it has the potential to reflect the level of voluntary intention and can be used to estimate the joint angle or torque [34-36]. In this study, EMG signal is also introduced to achieve joint torque estimation. As depicted in the 'FES-stimulated skeletal muscle' subsection, the active torque is the product of the contraction dynamics and the activation of the muscle. If the activation is induced by FES, the activation can be calculated using Equations 13, 14 , and 15; if the activation is voluntary-controlled, the EMG signal can be utilized as the index of the level of activation. The nonlinear relationship of the contraction dynamics of the muscle is approximated by BP neural networks. For the hip joint and the knee joint, there are six muscle groups involved. They are gluteus maximus (GM), iliopsoas (LP), biceps femoris short head (BFs), rectus femoris (RF), rectus lateralis (RL), and vastus medialis $(\mathrm{VM})$. 

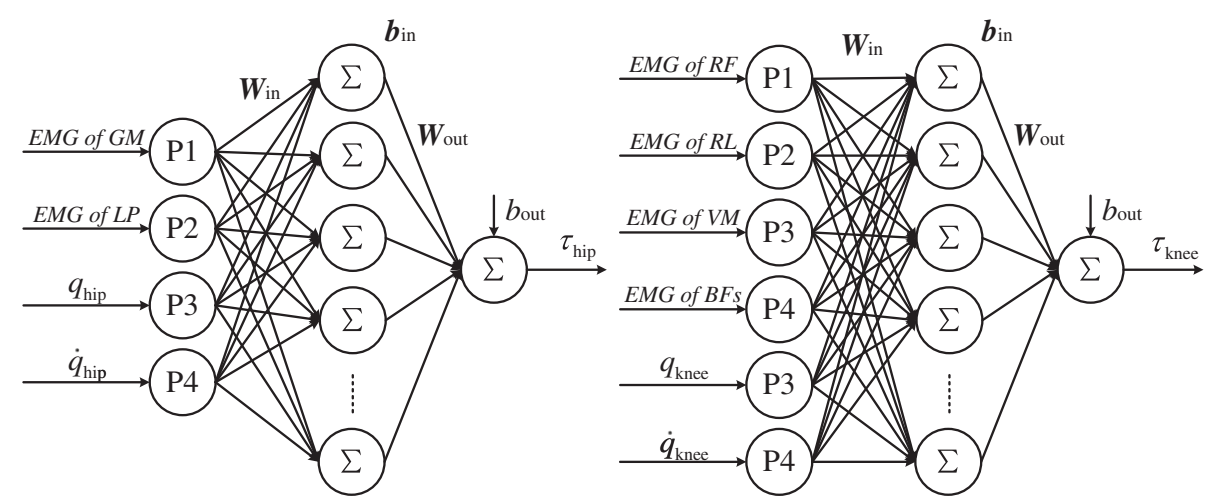

Figure 9 Neural network structures of the hip joint and the knee joint for voluntary torque estimation.

We construct two independent neural networks for the torque estimation of the hip joint and the knee joint. The structures of these two networks are shown in Figure 9. Each BP neural network has three layers, and the weight matrixes between the input layer, the hidden layer, and the output layer are adjusted using back propagation algorithm. Note that these EMG signals which served as the inputs of the neural networks are root mean square (RMS) EMG signals defined as

$$
\operatorname{RMS}(t)=\sqrt{\frac{1}{N} \sum_{i=t-N+1}^{t} y^{2}(i)}
$$

where RMS $(t)$ is the RMS EMG signal at time $t ; y(i)$ is the $i$ th sample of the raw EMG signal; $N$ is the length of sliding window which contains samples of $200 \mathrm{~ms}$ before time $t$.
The torques of the hip joint and the knee joint as well as the EMG signals are acquired during the robot performing the predefined trajectory, and at the same time, the subject makes random efforts. Ten groups of samples are collected, and leave-one-out cross validation process is carried out: nine groups of the samples are used for training and the rest for validation, then the data set for training and validation are changed; this procedure is executed ten times, which means that every group will be served as validation group for one time. The overall estimation error is the average error of each times.

Figure 10 shows the EMG signals and torques of the hip joint and the knee joint acquired during the subject making random efforts. The curves of the measured torques and the estimated torques on the hip joint and the knee joint are shown in Figure 11. The average RMS error
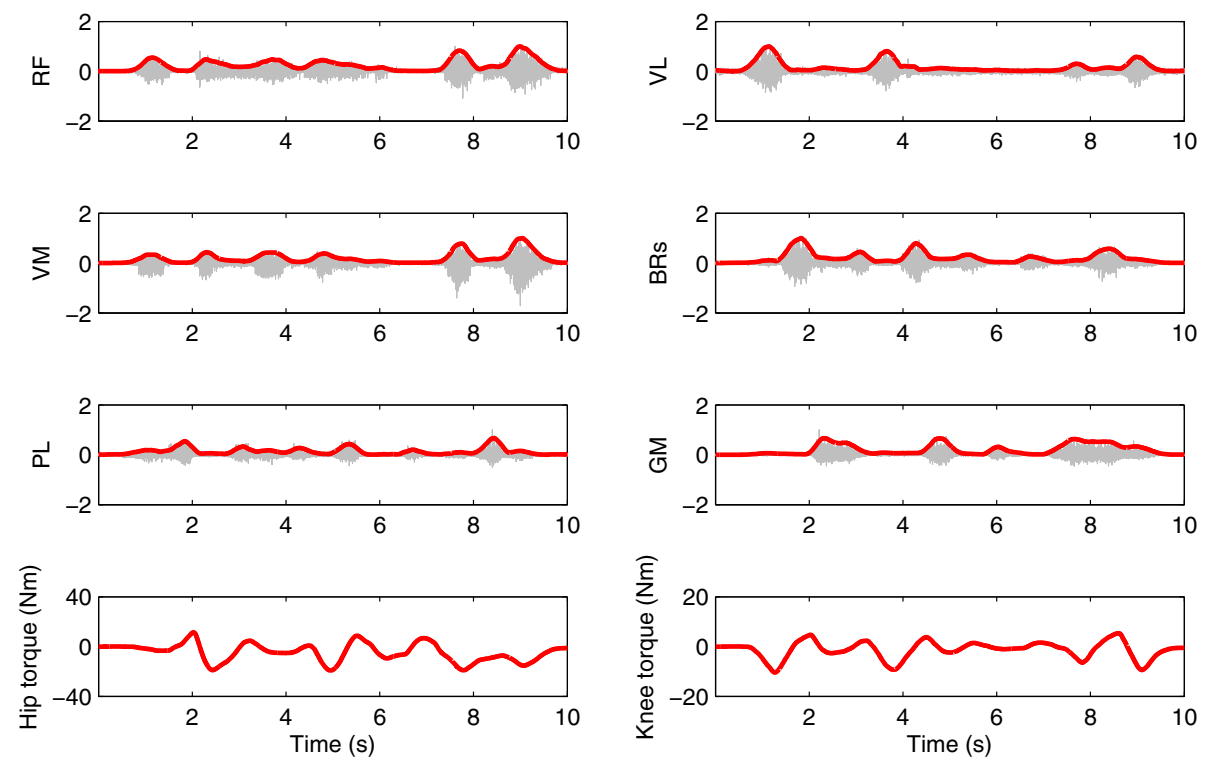

Figure 10 EMG signals and joint torques acquired during the subject making random efforts. Left, hip torque. Right, knee torque. 

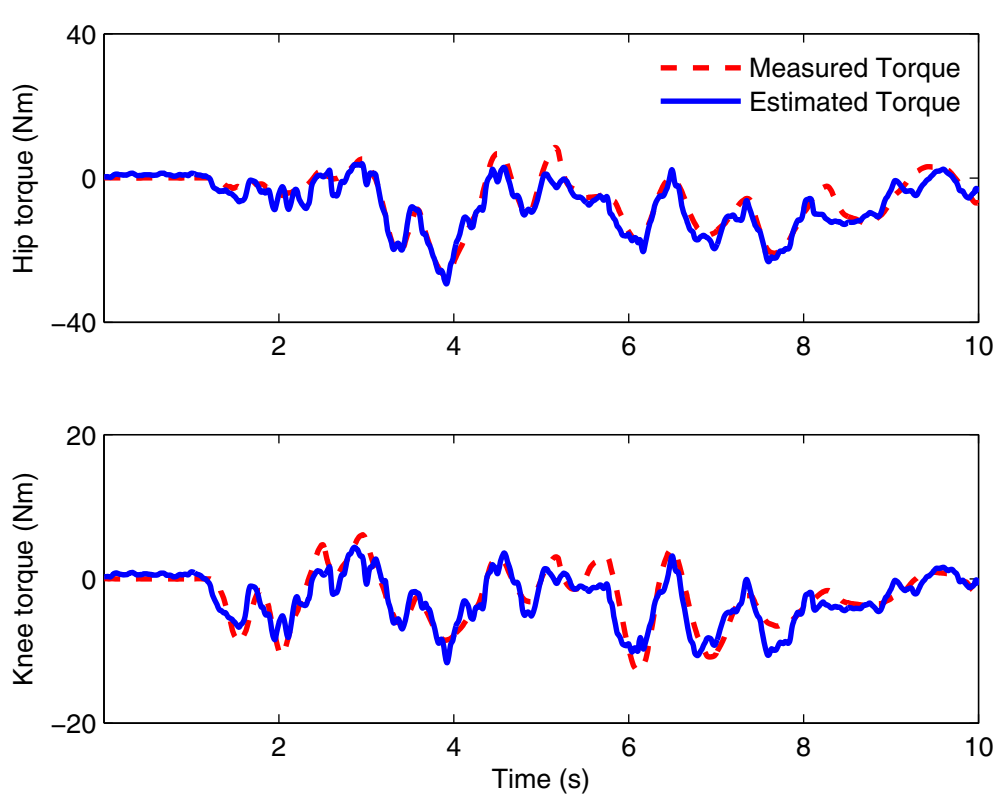

Figure 11 The curves of the measured and estimated torques on the hip knee joints. Top, hip torque. Bottom, knee torque.

is $0.8862 \mathrm{Nm}$ for the hip joint and $1.3424 \mathrm{Nm}$ for the knee joint. These errors are in an acceptable range, so the voluntary-controlled torque $\tau_{\mathrm{vol}}$ can be estimated using EMG signals and the proposed BP neural networks.

\section{Results}

\section{Simulation results}

The above control strategy is verified using Simulink toolbox of Matlab. The link lengths and the masses are set to be $l_{1}=0.5 \mathrm{~m}, l_{2}=0.45 \mathrm{~m}, m_{1}^{\mathrm{h}}=10 \mathrm{~kg}, m_{2}^{\mathrm{h}}=$ $4.5 \mathrm{~kg}, m_{1}^{\mathrm{r}}=13 \mathrm{~kg}$, and $m_{2}^{\mathrm{r}}=12 \mathrm{~kg}$. The parameters to construct the simulation models of gluteus maximus and quadriceps femoris are chosen according to [22,37]. For the leg press exercise, the patient is requested to accomplish the motion following a predefined trajectory substantially, and the predefined trajectory is a linear path with the start point set as $(0.5,0.1)$, and target point set as $(0.8,0)$ in the Cartesian space coordinates. The patient is encouraged to accomplish the task within about $10 \mathrm{~s}$.

The PI parameters for the speed controller described in Equation 19 are set as $K_{\mathrm{P}}=\operatorname{diag}(500,500)$ and $K_{\mathrm{I}}=$ $\operatorname{diag}(100,100)$. The stiffness and damping parameters in Equation 18 are set as $K_{\mathrm{d}}=\operatorname{diag}(60,60)$ and $B_{\mathrm{d}}=$ $\operatorname{diag}(30,30), \dot{x}_{\mathrm{d}}$ is set as zero, and $x_{\mathrm{d}}$ is defined as

$$
x_{\mathrm{d}}(t)=\left[\begin{array}{ll}
0.5+0.03 t \cos \theta & 0.1-0.03 t \sin \theta
\end{array}\right]^{\mathrm{T}}
$$

where $t$ represents time, and $\theta$ is the angle of the desired path to the horizontal.
To simulate the voluntary effort of the patient, random voluntary torques around the hip joint and the knee joint are implemented in the simulation, which is shown in Figure 12. In real situation, voluntary torque has to be estimated by means of the EMG signals, however, for simplicity reason, this step has been skipped. Note that the voluntary effort of the patient starts at $t=1 \mathrm{~s}$, and withdrawals at $t=6 \mathrm{~s}$.

Figure 13a shows the actual trajectory of the endpoint during the leg press exercise. The color of each point on the trajectory represents the speed of the endpoint at the very position. Hotter color indicates a relatively higher speed, and cooler color indicates a relatively lower speed. Virtual channel is also visualized in Figure 13a, with the predefined path at the center of the channel. Figure 13b is the zoom-in view of the trajectory for the first $2 \mathrm{~s}$. The muscular force which is the resultant force of voluntary force and FES-induced force is presented as a black arrow, pointing to the direction of the force, with the length denoting the strength. As the endpoint deviates from the predefined path, the component of force perpendicular to the path increases. This means the patient has to apply more force to counterbalance the 'virtual force' which drags the endpoint back to the predefined path according to the impedance mechanism, therefore reminding the patient of the correct direction of the excise. From the patient's perspective, it is much easier to press his leg towards the target point than towards the incorrect direction. As voluntary effort withdraws at $t=6 \mathrm{~s}$, the endpoint stops at $(0.74,0.02)$. Since the position error is modified by the mod factor in Equation 20 whose parameters are set as $a=-200, b=6$, the robot does not 


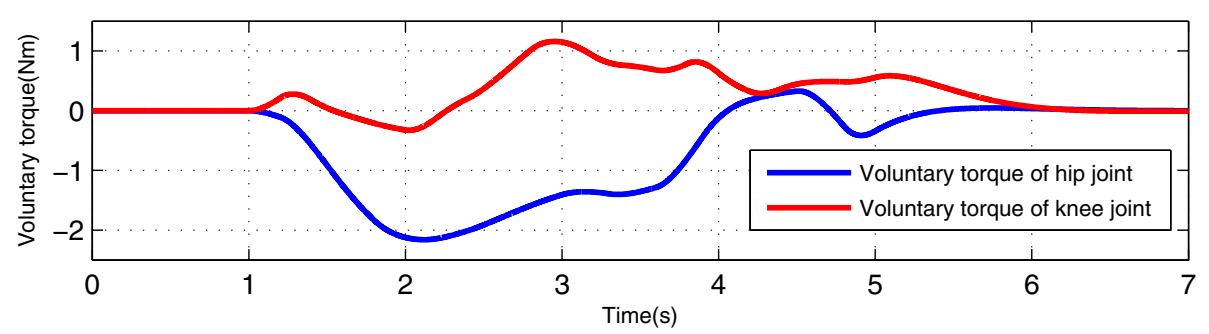

Figure 12 Voluntary torques generated by patient.

assist immediately. However, once the distance between the desired position and the actual position increases to a certain degree, the robot assists the patient to complete the task. The angular speeds of the hip joint and the knee joint are shown in Figure 14. When muscular force is applied on the robot, the robot begins to move (after $t=1 \mathrm{~s}$ ); when it is withdrawn, the speed of the robot drops to zero (after $t=6 \mathrm{~s}$ ). Followed by a waiting of about 4 $\mathrm{s}$, expecting the patient to make attempt again, however, in this simulation, if the patient does not take acting, the robot begins to assist and finally 'drag' the leg to reach the target point. The above results prove the first goal which is to create a compliance environment to inspire the voluntary effort of the patient is achieved.

The FES control is conducted first by determining the desired torque induced by FES. The idea is to amplify the voluntary effort towards the target point. Using Equation 21, the force component of the voluntary effort parallel to the predefined trajectory is calculated, amplified, and then translated to the desired active torque for FES control in joint space. The blue arrows in Figure 13b represent the desired active force expected being induced by FES. All of them point to the same direction, the direction of the target point. This indicates that the FES assistance is not only proportional to the voluntary effort but also provided in the correct direction. The blue dash line in Figure 13a shows the trajectory of the endpoint when the same voluntary effort is made, but this time, no FES assistance is applied. The comparison of these two trajectories verifies the contribution of FESinduced torque which assists the patient during the leg press task by enhance the 'correct part' of the voluntary effort.

The FES control contains two parts: feedforward control and feedback control. For the training of the neural network-based inverse muscle models, samples are collected at pre-training stage, in which the muscles are applied with FES of random intensity while the robot is running in the predefined trajectory. The pulse width $d$ of the FES uniformly distributed on the interval [ $\left[\begin{array}{ll}0 & 500\end{array}\right]$ $\mu \mathrm{s}$. Off-line training process is conducted with 5,014 sampling, and both neural networks for gluteus maximus and quadriceps femoris converge after 250 iterations with the mean square errors below $5.47 \mu$ s for gluteus maximus (a)

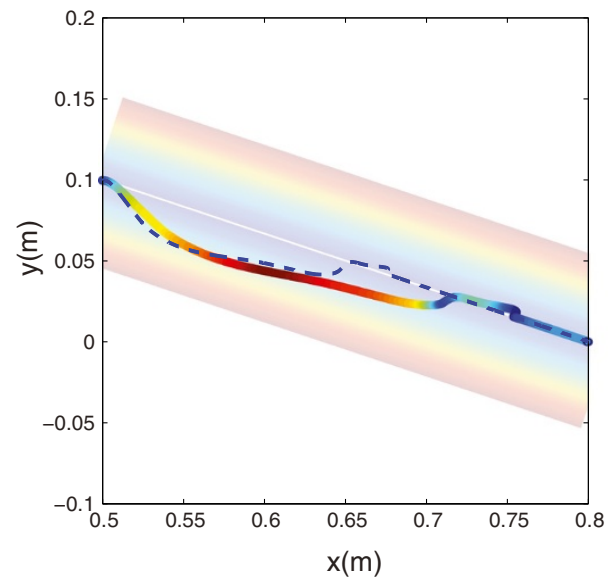

(b)

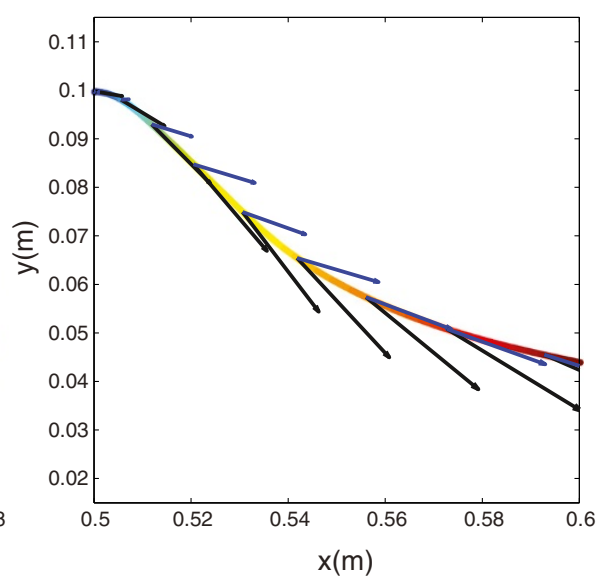

Figure 13 Trajectory of the endpoint in Cartesian space. (a) Entire trajectory of the endpoint with the color indicating the speed and (b) zoom-in view of the trajectory for the first $2 \mathrm{~s}$. 
(a)

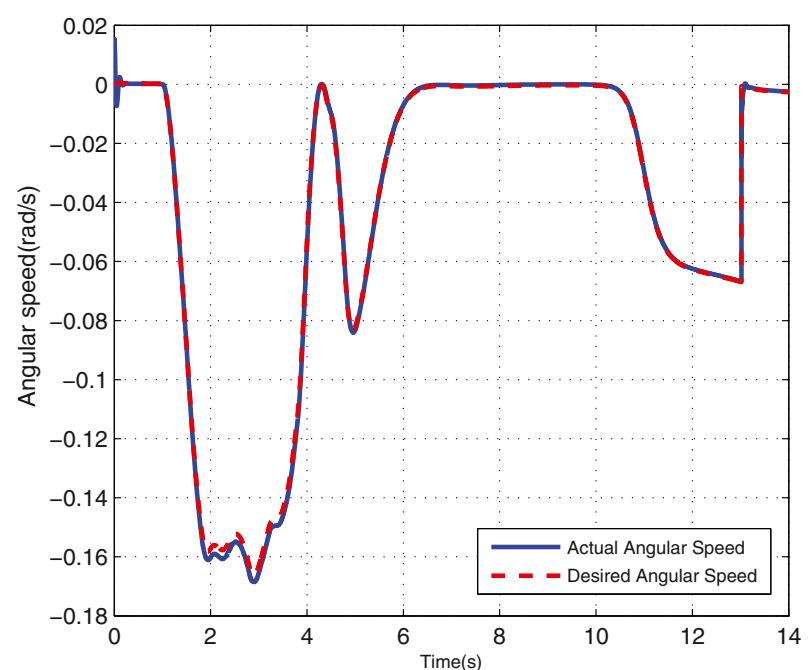

(b)

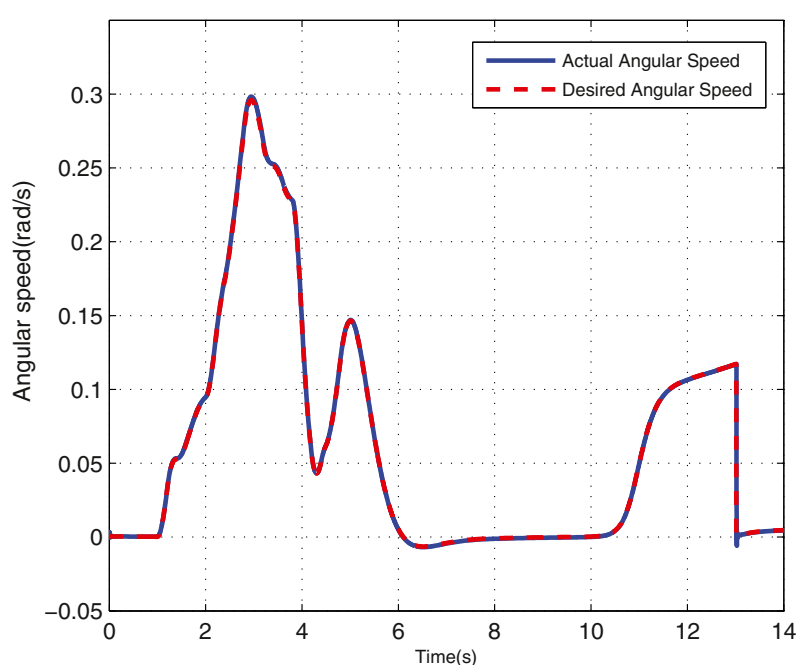

Figure 14 Speed tracking performance of the robot. (a) Curve of the angular velocity of the hip joint. (b) Curve of the angular velocity of the knee joint.

and $3.98 \mu$ s for the quadriceps femoris. The effectiveness of the inverse model is shown in Figure 15. Random desired active torque ( 0 to $3 \mathrm{Nm}$ for quadriceps femoris and -3 to $0 \mathrm{Nm}$ for gluteus maximus) is input into the inverse model when the robot is running on the predefined trajectory for validation, and the outputs are used to set the intensity of the FES which is applied on the muscles. The approximation of the profiles for desired torque and active torque in both Figure 15a,b shows that the inverse model is capable of outputting the proper pulse width for FES to generate the desired torque. As described in the former FES control section, $\hat{\tau}_{\mathrm{fes}}$ is implemented as the estimation of actual active torque, and the error between $\tau_{\text {dfes }}$ and $\hat{\tau}_{\text {fes }}$ serves as the input of feedback controller described in Equation 22 with the PD parameter set as $K_{\mathrm{P}}=\operatorname{diag}(3,000 ; 3,000), K_{\mathrm{D}}=\operatorname{diag}(400,400)$. (a)

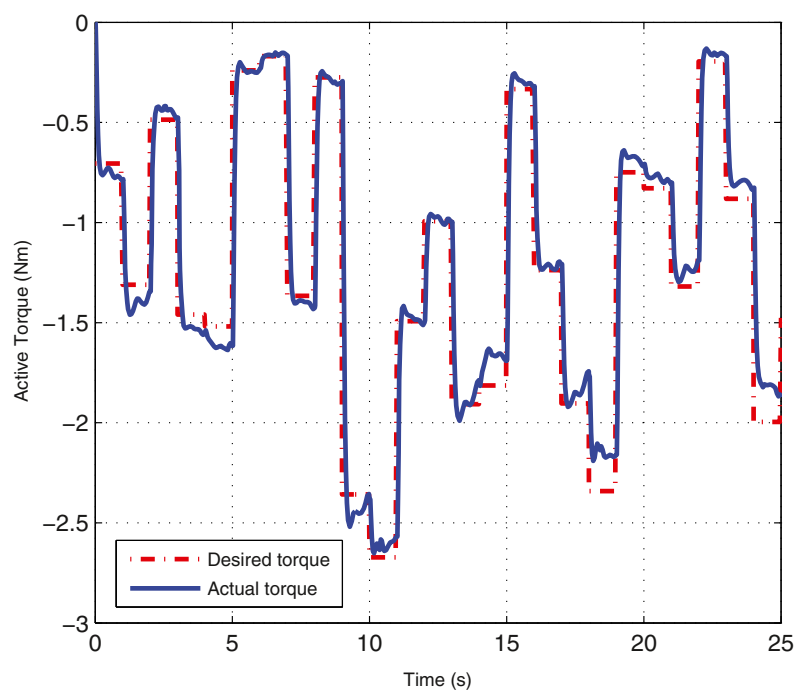

(b)

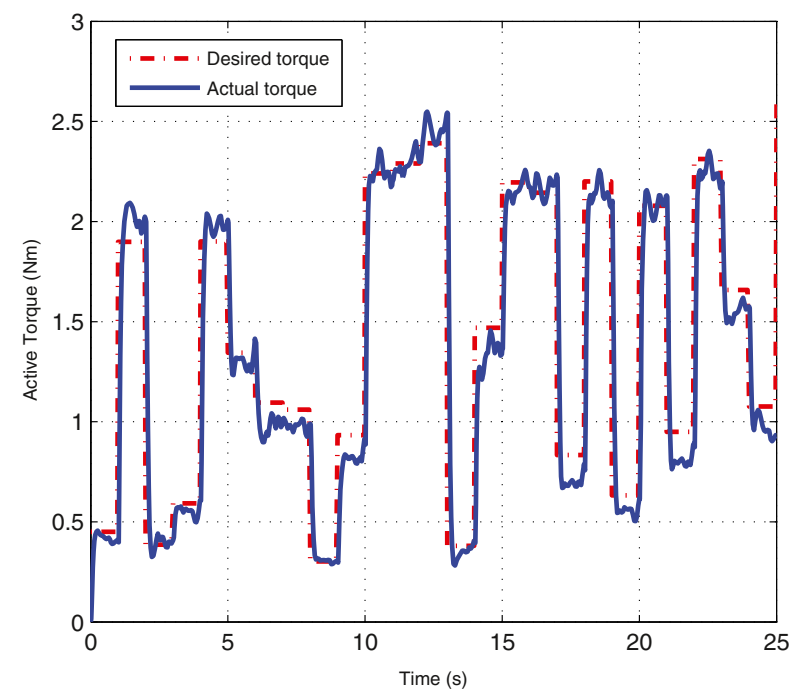

Figure 15 Results of the inverse muscle model for generating desired active torque. Validation results of the inverse models for the gluteus maximus (a) and quadriceps femoris (b) 
(a)

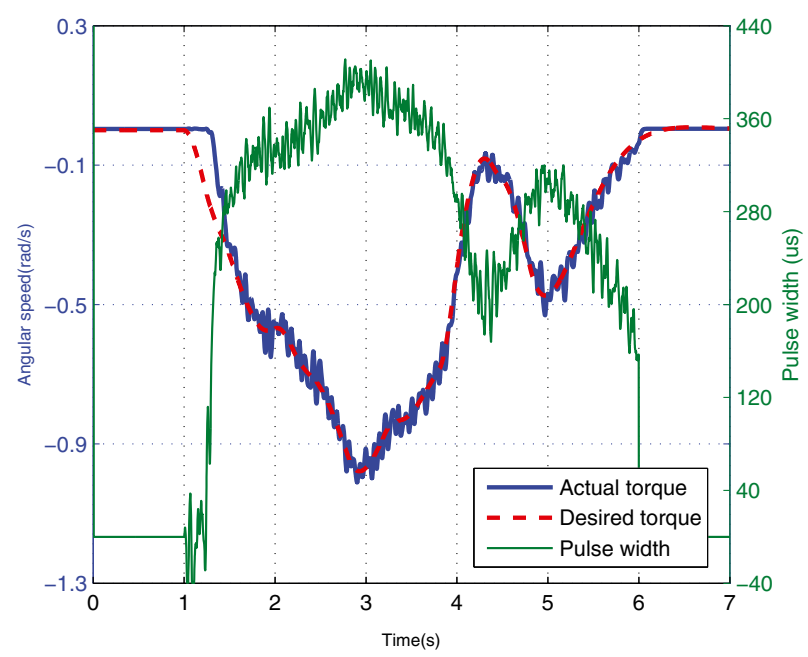

(b)

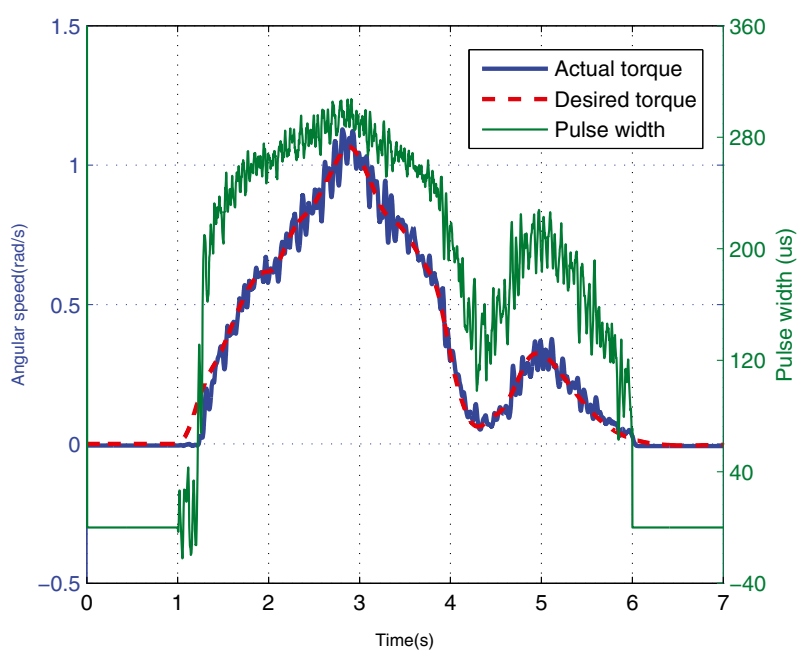

Figure 16 FES-induced torque tracking performance. Tracking performance of the gluteus maximus (a) and quadriceps femoris (b).

Figure 16 shows the desired torque $\tau_{\mathrm{dfes}}$ and the estimated active torque $\hat{\tau}_{\text {fes }}$ produced by the muscle contraction response to FES. The green line is the pulse width calculated by the FES controller, and FES whose intensity depends on this pulse width is applied on the muscle to generate the desired active torque. The profiles of the red line and the blue line indicate that the error between the desired torque and the actual active torque is in an acceptable range which proves that the FES controller is capable of inducing desired active torque so that a precise assistance through FES can be delivered to the patient.

\section{Experiment results}

The proposed control strategy is also verified by the experiment conducted on iLeg lower limb rehabilitation robot. The method described in 'FES-induced torque estimation' subsection is utilized to obtain voluntary torque $\tau_{\mathrm{vol}}$ and FES-induced torque $\tau_{\text {fes. }}$. An SCI subject (male, age 65) and a healthy subject (male, age 28) are invited to participate in the experiment. The SCI patient has been injured in T11 for 16 months, and he remains with partial muscle force. The speed control of the robot is carried out by the PID controller of the motor drivers. The stiffness (a)

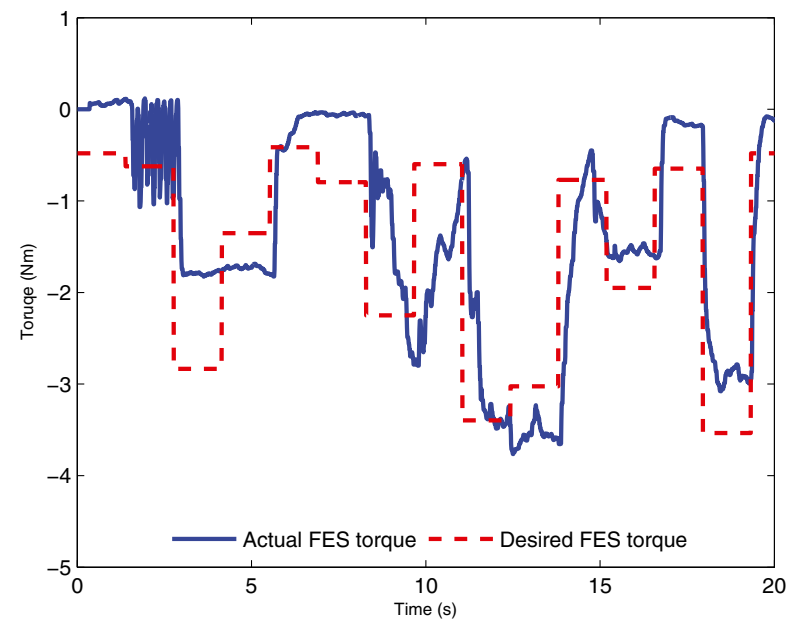

(b)

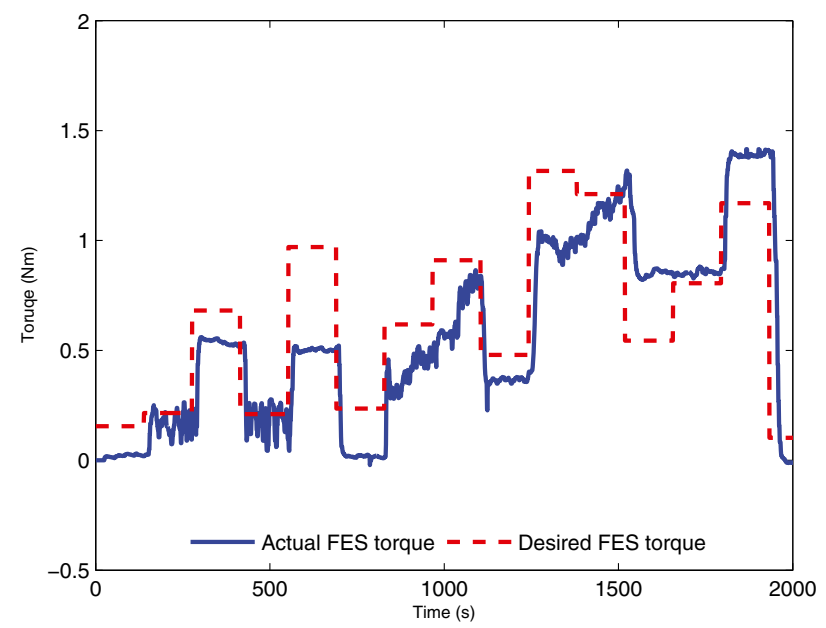

Figure 17 Results of the inverse muscle model for generating desired active torque (SCl subject). Validation results of the inverse models for the gluteus maximus (a) and quadriceps femoris (b). 
(a)
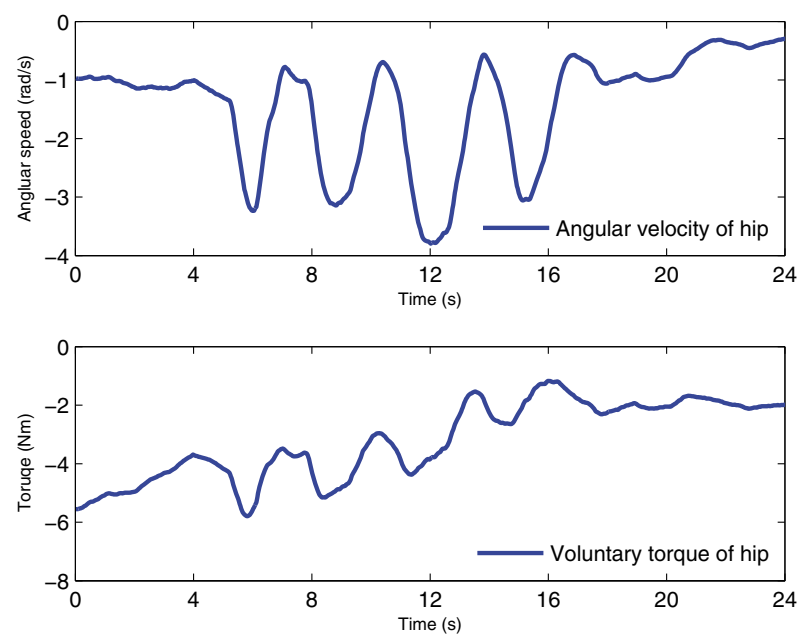

(b)
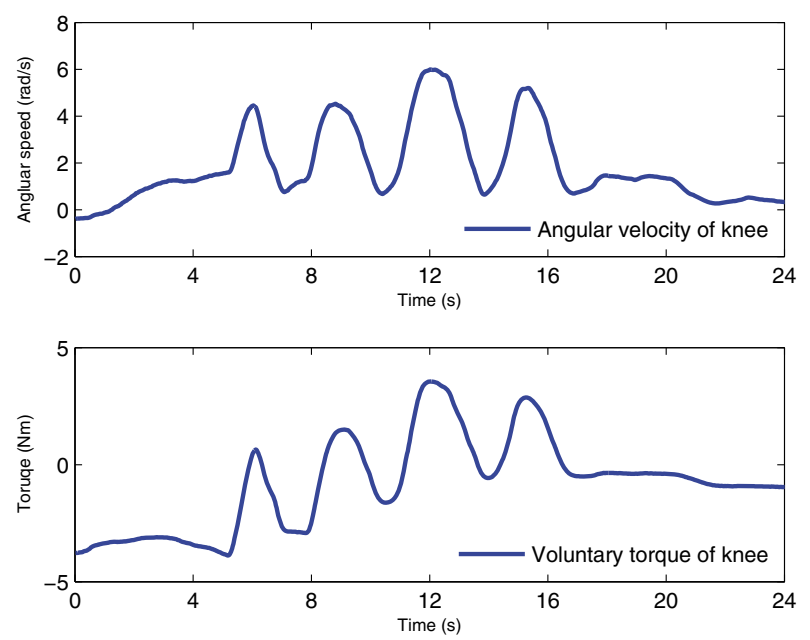

Figure 18 Curves of the voluntary torques and angular velocities of each joint (SCl subject). (a) Hip. (b) Knee.

and damping parameters in Equation 18 are set as $K_{\mathrm{d}}=$ $\operatorname{diag}(2,500 ; 2,500)$ and $B_{\mathrm{d}}=\operatorname{diag}(2,000 ; 2,000)$. $\dot{x}_{\mathrm{d}}$ is set as zero. Considering that the SCI subject's motion ranges of his lower limb are limited, we decrease the length of the predefined trajectory, and set the start point to be $(0.5,0.1)$ and the target point to be $(0.7,0)$.

At the pre-training stage, the tolerance of the subject applied with FES is tested. For each stimulated muscle, the pulse width of FES is firstly fixed at $700 \mu \mathrm{s}$, and the amplitude of FES is increased from $15 \mathrm{~mA}$ until obvious muscle contraction being observed. Then the amplitude of the FES is fixed during training, and the intensity of FES is regulated by adjusting the pulse width through FES controller. To construct the BP neural networks which serve as the FES feedfoward controller, the muscles are applied with FES of random intensity while the robot running in the predefined trajectory. The pulse width $d$ of the FES uniformly distributed on the interval [150 500] $\mu$ s. (a)

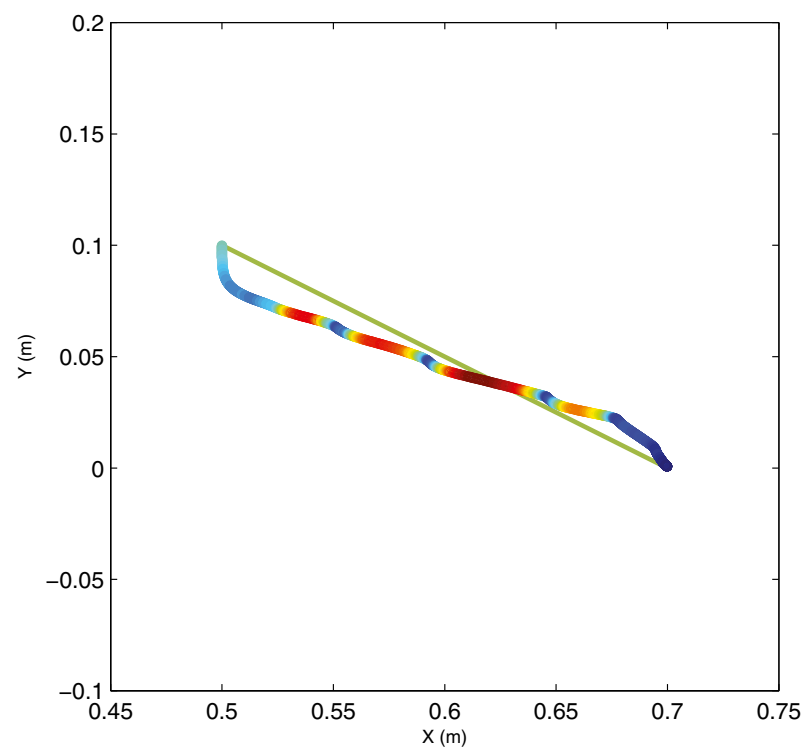

(b)

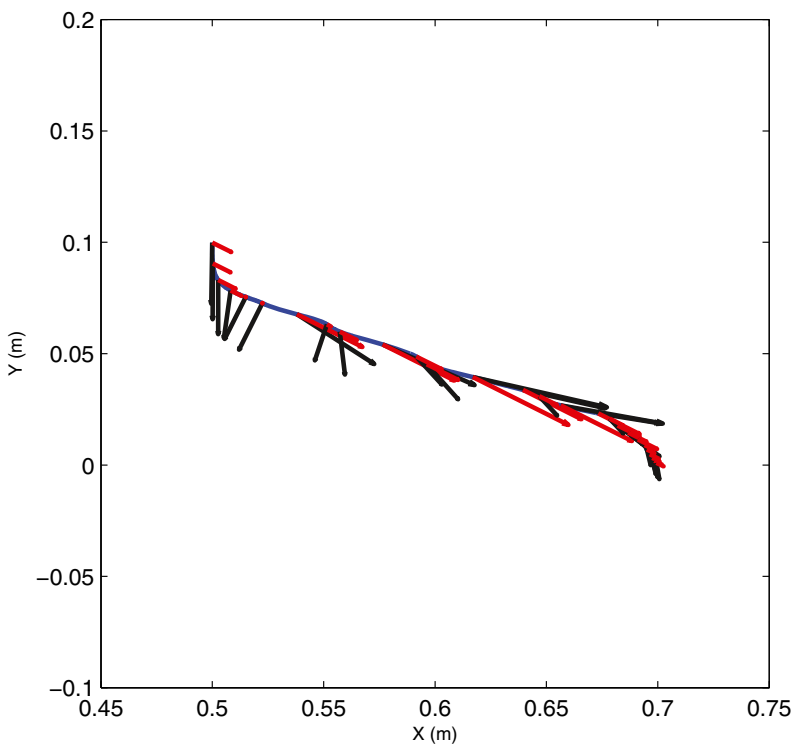

Figure 19 Trajectory of the endpoint in Cartesian space (SCI subject). (a) Entire trajectory of the endpoint with the color indicating the speed. (b) Voluntary forces of the subject and the FES-assisted forces using black arrows and red arrows, respectively. 

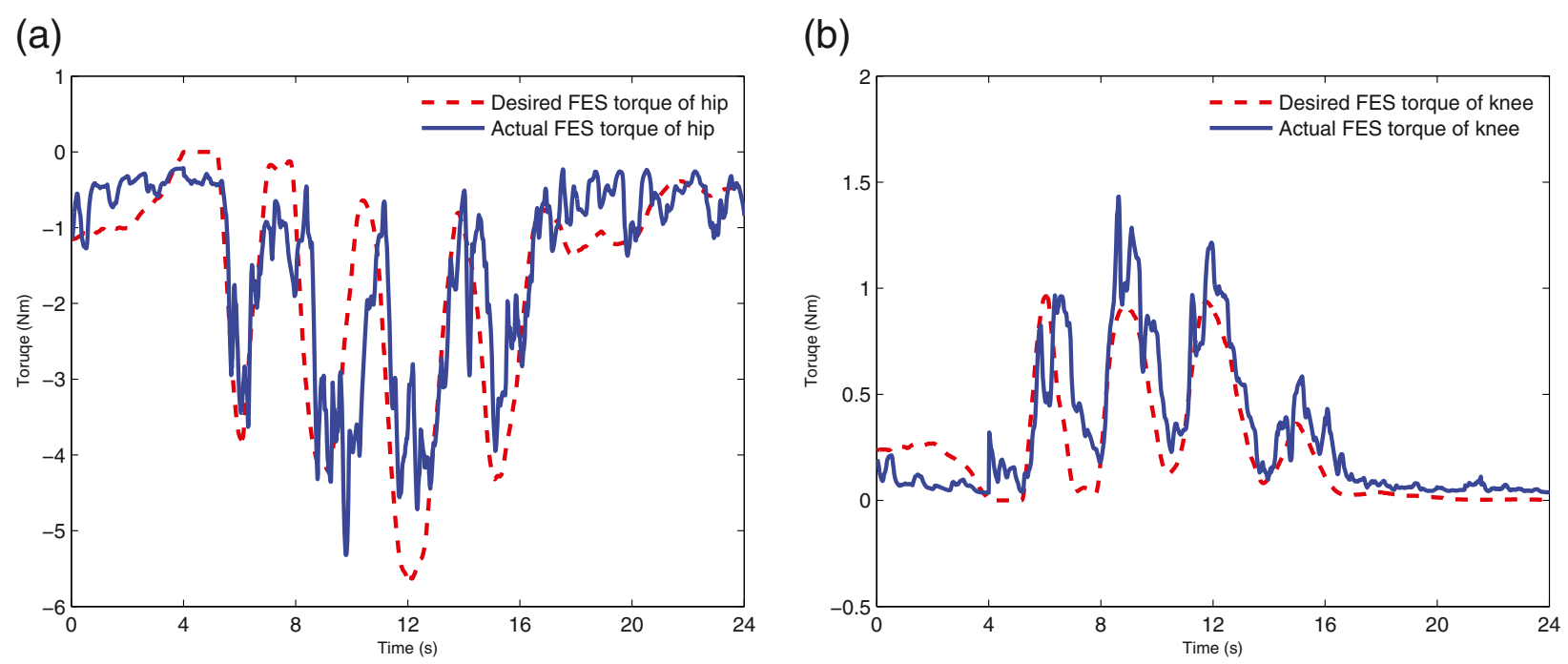

Figure 20 FES-induced torque tracking performance ( $\mathrm{SCl}$ subject). Tracking performance of the gluteus maximus (a) and quadriceps femoris (b)

The effectiveness of the inverse model is shown in Figure 17. Random desired active torques ( 0 to $1.5 \mathrm{Nm}$ for quadriceps femoris and -3.5 to $0 \mathrm{Nm}$ for gluteus maximus) are input into the inverse model when the robot is running on the predefined trajectory, and the outputs are used to set the intensities of the FES applied on the muscles. Compared to the simulation results, the muscle delay is relatively serious and the results are not so satisfactory which reflects that the FES-induced muscle contraction is unstable. However, the error is acceptable and can be eliminated by the PD feedback controller.

Figure 18 shows the curves of voluntary torques estimated by EMG signals and the angular velocities of each joint. As the voluntary torques vary, the angular velocities of each joint change accordingly, which follows the principle of impedance control. The trajectory of the endpoint is shown in Figure 19. There are four obvious efforts represented by four red sections in Figure 19a and four peaks in the curve of angular velocity in Figure 18. As the same as in Figure 13b, the FES-assisted forces and voluntary forces are drawn as several arrows in Figure 19b from which the same conclusion can be drawn: the FES assistance is not only proportional to the voluntary effort but is also provided in the correct direction. The tracking performances of the FES controller which is the combination of a feedforward controller and a PD feedback controller (a)
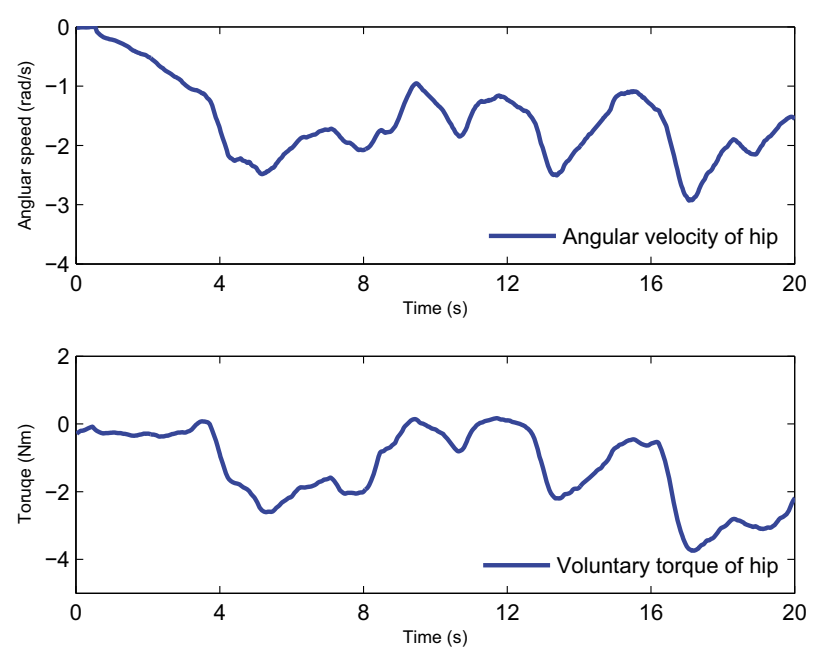

(b)
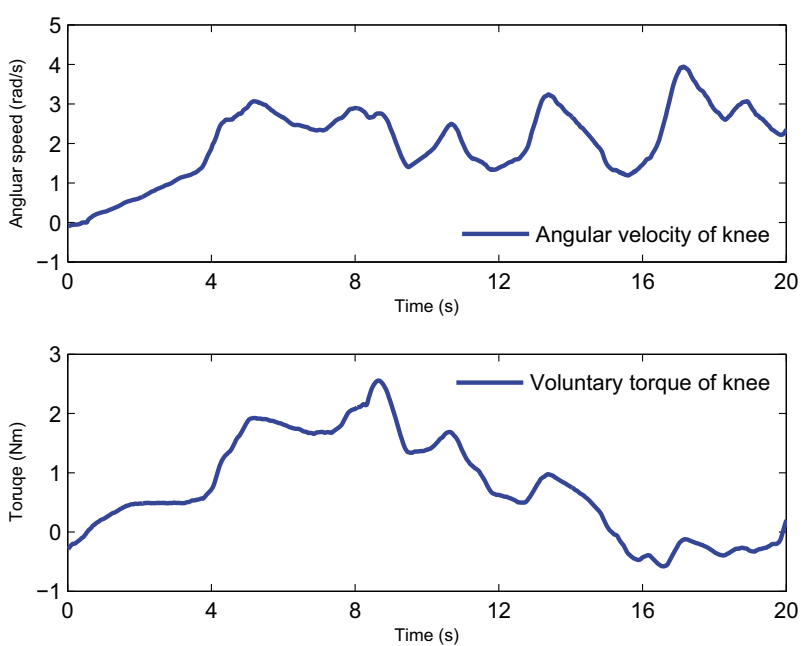

Figure 21 Curves of the voluntary torques and angular velocities of each joint (healthy subject). (a) Hip. (b) Knee. 
(a)

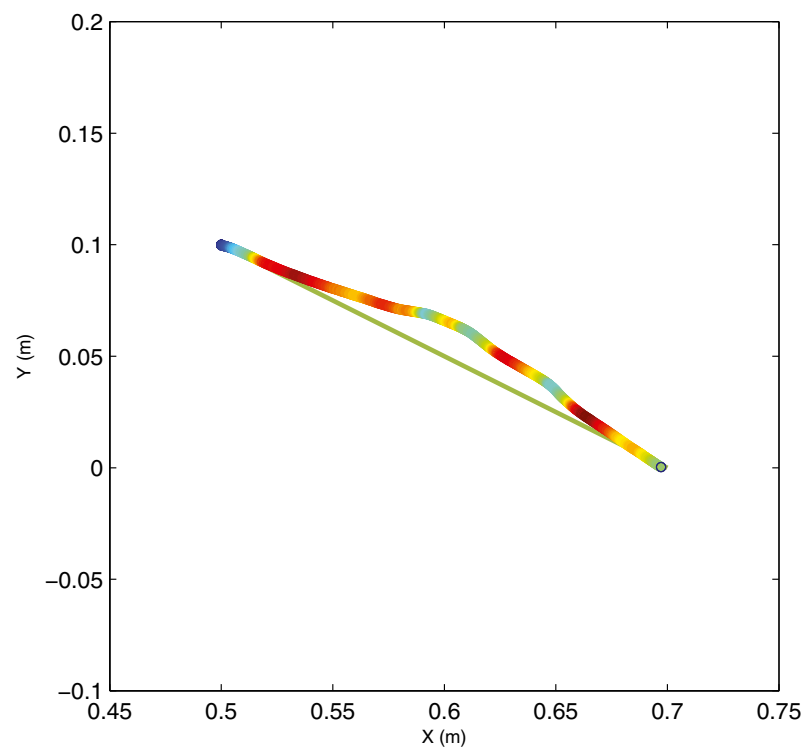

(b)

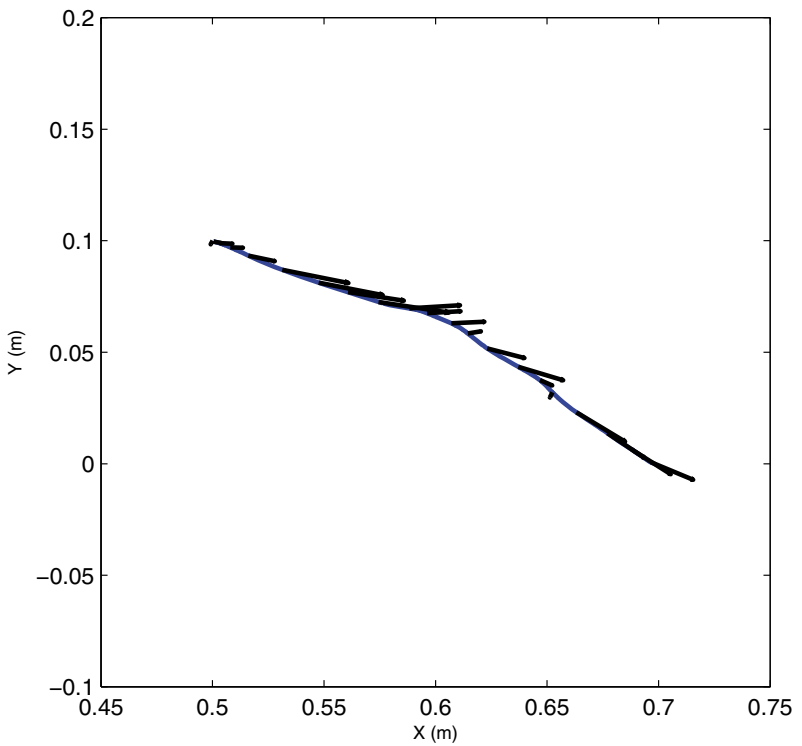

Figure 22 Trajectory of the endpoint in Cartesian space (healthy subject). (a) Entire trajectory of the endpoint with the color indicating the speed. (b) Voluntary forces of the subject using red arrows.

are represented in Figure 20. Even though the tracking is not as precise as in the simulation, these results indicate the FES controller is capable of generating desired FES-induced torque to apply assistance to the patient.

One healthy subject is also involved in this experiment. However, during the training process, no FES assist is applied on the healthy subject, only the effect of the impedance control is validated. Figures 21 and 22 are the curves of the voluntary torques and the angular velocities of each joint, the trajectory of the endpoint, respectively. Besides the similar conclusion that we can obtain from Figures 18 and 19, we can also notice that the voluntary (a)

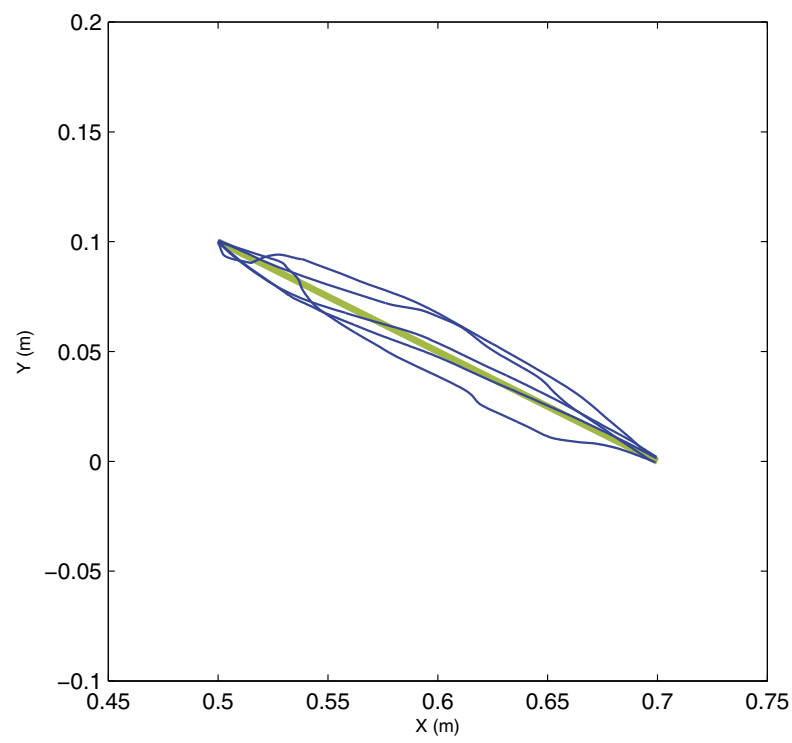

(b)

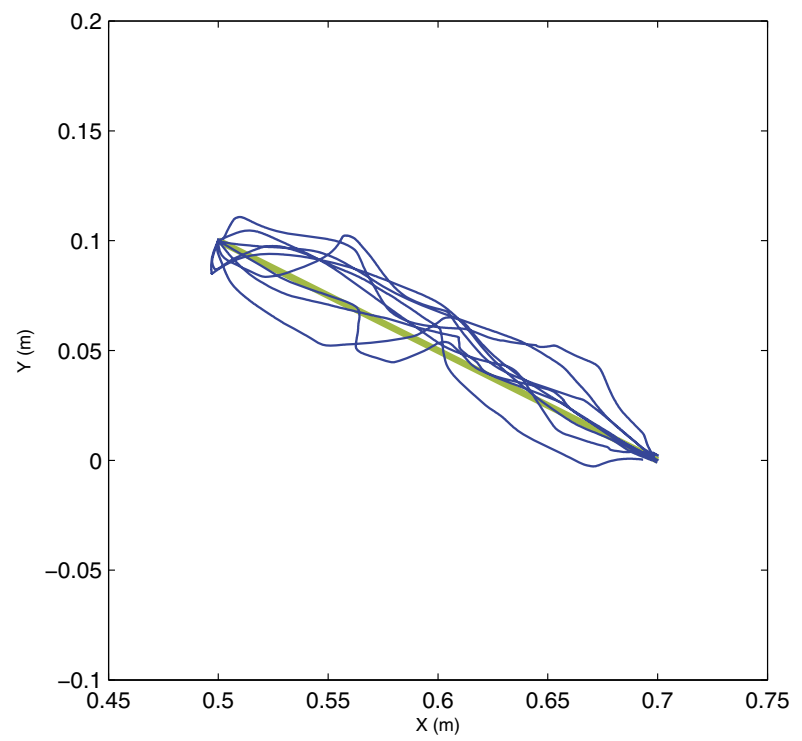

Figure 23 Comparison of the trajectories of the endpoint. The end-point trajectories of the healthy subject (a) and the SCI subject (b) in several leg press trials. 


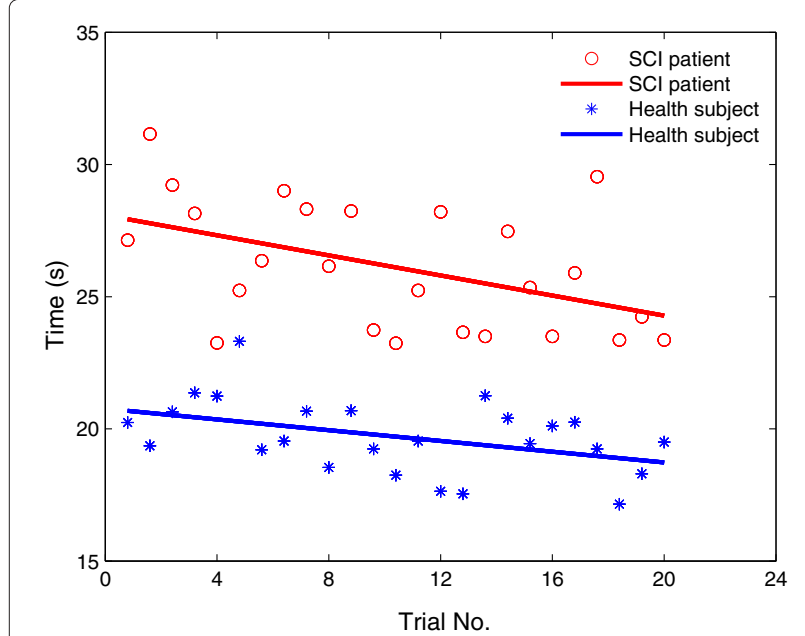

Figure 24 The time consumption for 20 leg press trials.

efforts are smoother compared with the effort made by the SCI subject, resulting a smooth trajectory of the endpoint.

The end-point trajectories of several trials for both the SCI subject and the healthy subject are drawn in Figure 23. The end-point trajectories of the healthy subject are much smoother than the end-point trajectories of the SCI subject, reflecting that the SCI subject's controlling ability of his lower limb is insufficient.

Figure 24 represents the time consumption for $20 \mathrm{leg}$ press trials. As we can see from Figure 24, due to lack of muscle strength, the average time consumption of the SCI subject is more than that of the healthy subject. We also notice that as the trial number increases, the time consumption decreases. This phenomenon indicates that through repetitive training, both subjects gradually adjust the patten of making effort so that the performance of the leg press exercise is improved.

\section{Conclusions}

In this paper, an FES-assisted training strategy combined with impedance control for the lower limb rehabilitation robot is presented and the controller used to accomplish this task is developed, aiming to achieve two goals. The first one is to create a compliance environment to inspire the voluntary effort of the patient, and the second one is to provide assistance through FES rather than purely through robot. Impedance control is ideal for establishing active compliance of the robot, and through the mechanism of impedance control, the patient has to make effort to accomplish the task rather than passively waiting the assistance from the robot so that it increases the participation of the patient's voluntary effort. Two muscle groups are stimulated with FES to provide extra assistance to the patient during the training process. The intensity of the FES is properly chosen so as to induce the desired active torque which is proportional to the voluntary effort of the patient. The control of active torque of FES-stimulated muscle is achieved using the combination of a neural network-based feedforward controller and a PD feedback controller which have been proven to be competent for this task. The dynamic parameters of the leg-robot hybrid system are estimated using system identification method; the voluntary torques are also estimated according to the EMG signals acquired from the related muscle groups. These two methods are utilized to obtain the actual FES-induced torque so that the FES control can be accomplished. Both simulation conducted in Matlab and experiments conducted in iLeg reveal satisfactory results, from which the feasibility and effectiveness of the proposed control strategy are verified. In the future, more SCI and stroke subjects will be recruited to carry out a 3 to 6-month training session. During this follow up study, we will evaluate whether the proposed control strategy is capable of improving the rehabilitation effects.

\section{Abbreviations}

BFI: biceps femoris long head; BFs: biceps femoris short head; DOF: degrees of freedom; EMG: electromyography; FES: functional electrical stimulation; GM: gluteus maximus; RF: rectus femoris; RL: rectus lateralis; RMS: root mean square; VM: vastus medialis.

\section{Competing interests}

The authors declare that they have no competing interests.

\section{Authors' contributions}

YC conceived the concept of the study, participated in acquisition and analysis of data, and drafted the manuscript. HJ participated in the development of the robot platform. LP participated in acquisition of data and made contributions to the revision of the draft. $\mathrm{ZH}$ made contributions to the revision of the draft. All authors read and approved the final manuscript.

\section{Acknowledgements}

This work was supported in part by the National Natural Science Foundation of China (Grants 61225017, 61175076) and the International S\&T Cooperation Project of China (Grant 2011DFG13390).

Received: 4 May 2014 Accepted: 28 May 2014

Published online: 02 October 2014

\section{References}

1. Marino R, Barros T, Biering-Sorensen F, Burns S, Donovan W (2003) International standards for neurological classification of spinal cord injury. J Spinal Cord Med 26:50-56

2. Sims NR, Muyderman H (2010) Mitochondria, oxidative metabolism and cell death in stroke. Biochim Biophys Acta - Molecular Basis of Disease 1802(1):80-91

3. Freeman C, Tong D, Meadmore K, Hughes AM, Rogers E, Burrideg J (2012) FES based rehabilitation of the upper limb using input output linearization and ILC. In: Proceedings of the 2012 American Control Conference, pp 4825-4830. http://ieeexplore.iee.org/Xplore/home.jsp

4. Lo HS, Xie SQ (2012) Exoskeleton robots for upper-limb rehabilitation: state of the art and future prospects. Med Eng Phys 34:261-268

5. Diaz I, Gil JJ, Sanchez E (2011) Lower-limb robotic rehabilitation: literature review and challenges. J Robot 2011:1-10

6. Crespo LM, Reinkensmeyer DJ (2009) Review of control strategies for robotic movement training after neurologic injury. J Neuroeng Rehabil $6: 20$

7. Jung S, Hsia T (2000) Robust neural force control scheme under uncertainties in robot dynamics and unknown environment. IEEE Trans Ind Electron 47(2):403-412 
8. Barreca S, Wolf S, Fasoli S, Bohannon R (2003) Treatment interventions for the paretic upper limb of stroke survivors: a critical review. Neurorehabil Neural Repair 17(4):220-226

9. Kwakkel G, Kollen BJ, Wagenaar RC (1999) Therapy impact on functional recovery in stroke rehabilitation: a critical review of the literature. Physiotherapy 85(7):377-391

10. Fiering-Sorensen F, Andersen OT, Hansen SD (1997) Functional neuromuscular stimulation controlled by surface electromyographic signals produced by volitional activation of the same muscle: adaptive removal of the muscle response from the recorded EMG-signal. IEEE Trans Rehabil Eng 5(2):195-206

11. Giuffrida JP, Crago PE (2001) Reciprocal EMG control of elbow extension by FES. IEEE Trans Neural Syst Rehabil Eng 9(4):338-345

12. Duschau-Wicke A, von Zitzewitz J, Caprez A, Lunenburger L, Riener R (2010) Path control: a method for patient-cooperative robot-aided gait rehabilitation. IEEE Trans Neural Syst Rehabil Eng 18:38-48

13. Kosuge K, Fujisawa Y, Fukuda T (1993) Mechanical system control with man-machine-environment interactions. In: Proceedings of the 1993 IEEE international conference on robotics and automation, pp 239-244. http://ieeexplore.ieee.org/Xplore/home.jsp

14. Tsumugiwa T, Yokogawa R, Hara K (2002) Variable impedance control based on estimation of human arm stiffness for human-robot cooperative calligraphic task. In: Proceedings of the IEEE international conference on robotics and automation, pp 644-650. http://ieeexplore. ieee.org/Xplore/home.jsp

15. Emken JL, Reinkensemeyer DJ (2005) Robot-enhanced motor leaning accelerating internal model formation during locomotion by transient dynamic amplification. IEEE Trans Neural Syst Rehabil Eng 13:33-39

16. Lynch CL, Popovic MR (2012) A comparison of closed-loop control algorithms for regulating electrically stimulated knee movements in individual with spinal cord injury. IEEE Trans Neural Syst Rehabil Eng 20(4):539-548

17. Kroon JR, IJzerman MJ, Chae J, Lankhorst GJ, Zilvold G (2005) Relation between stimulation characteristics and clinical outcome in studies using electrical stimulation to improve motor control of the upper extremity in stroke. J Rehabil Med 37:65-74

18. Metrailler P, Brodard R, Clavel R, Frischknecht R (2006) Closed loop electrical muscle stimulation in spinal cord injured rehabilitation. Mediter J Phys Rehabil Med 42(4):72-83

19. Freeman $C T$, Hughes $A M$, Burridge $J H$, Chappell $P H$, Lewin PL, Rogers $E$ (2009) A robotic workstation for stroke rehabilitation of the upper extremity using FES. Med Eng Phys 31:364-373

20. Rushton DN (2003) Functional electrical stimulation and rehabilitation an hypothesis. Med Eng Phys 25:75-78

21. Riener R, Edrich T (1999) Identification of passive elastic joint moments in the lower extremities. J Biomech 32:539-544

22. Riener R, Fuhr T (1998) Patient-driven control of FES-supported standing up: a simulation study. IEEE Trans Rehabil Eng 6(2):113-124

23. Ferrarin M, Palazzo F, Riener R, Quintern J (2001) Model-based control of FES-induced single joint movements. IEEE Trans Neural Syst Rehabil Eng 9(3):245-257

24. Almeida F, Lopes A, Abreu P (1999) Force-impedance control: a new control strategy of robotic manipulators. In: Recent advances in mechatronics. Springer, Singapore, pp 126-137

25. Anam K, Al-Jumaily AA (2012) Active exoskeleton control systems: State of the art. Procedia Eng 41:988-994

26. Aguirre-Ollinger G, Colgate JE, Peshkin MA, Goswami A (2007) Active-impedance control of a lower-limb assistive exoskeleton. In: Proceedings of the IEEE 10th international conference on rehabilitation robotics, pp 188-195. http://ieeexplore.ieee.org/Xplore/home.jsp

27. Yang Y, Wang L, Tong J, Zhang L (2006) Arm rehabilitation robot impedance control and experimentation. In: Proceedings of the IEEE international conference on robotics and biomimetics, pp 914-918. http://ieeexplore.ieee.org/Xplore/home.jsp

28. Zhang L, Sun H, Li C (2010) Experiment study of impedance control on horizontal lower limbs rehabilitation robot. In: Proceedings of the 2010 IEEE international conference on information and automation, pp 1421-1425. http://ieeexplore.ieee.org/Xplore/home.jsp

29. Lynch CL, Popovic MR (2008) Functional electrical stimulation. Contr Syst Mag 28(2):40-50
30. Denève A, Moughamir S, Moughamir A, Zaytoon J (2008) Control system design of a 3-DOF upper limbs rehabilitation robot. Comput Methods Programs Biomed 89(2):202-214

31. Swevers J, Ganseman C, Schutter JD, Brussel HV (1996) Experimental robot identification using optimised periodic trajectories. Mech Syst Signal Process 10(5):561-577

32. Wu W, Zhu S, Wang X, Liu H (2012) Closed-loop dynamic parameter identification of robot manipulators using modified fourier series. Int J Adv Robotic Syst 9:561-577

33. Kamen G, Gabriel DA (2010) Essentials of electromyography. Human Kinetics, Champaign

34. Sartori M, Reggiani M, Pagello E, Lloyd DG (2012) Modeling the human knee for assistive technologies. IEEE Trans Biomed Eng 59(9):2642-2649

35. Clancy EA, Liu L, Liu P, Moyer DVZ (2012) Identification of constant-posture EMG-torque relationship about the elbow using nonlinear dynamic models. IEEE Trans Biomed Eng 59(1):205-212

36. Clancy EA, Bouchard S, Rancourt D (2001) Estimation and application of emg amplitude during dynamic contractions. IEEE Eng Med Biol 20(6):47-54

37. Delp SL (1990) Surgery simulation: a computer graphics system to analyze and design musculoskeletal reconstructions of the lower limb. Ph.D. Dissertation

doi:10.1186/s40638-014-0002-7

Cite this article as: Chen et al:: The FES-assisted control for a lower limb rehabilitation robot: simulation and experiment. Robotics and Biomimetics 2014 1:2.

\section{Submit your manuscript to a SpringerOpen ${ }^{\circ}$ journal and benefit from:}

- Convenient online submission

Rigorous peer review

- Immediate publication on acceptance

- Open access: articles freely available online

- High visibility within the field

Retaining the copyright to your article

Submit your next manuscript at $>$ springeropen.com 